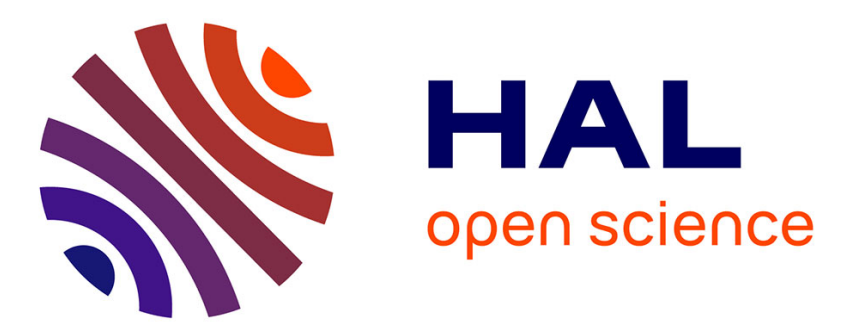

\title{
Au-delà de Kyoto: enjeux d'équité et d'efficacité dans la négociation sur le changement climatique
}

Odile Blanchard, Patrick Criqui, Michel Trommetter, Laurent Viguier

\section{To cite this version:}

Odile Blanchard, Patrick Criqui, Michel Trommetter, Laurent Viguier. Au-delà de Kyoto: enjeux d'équité et d'efficacité dans la négociation sur le changement climatique. Economie et Prévision, 2000, 143-144, pp.15-36. halshs-00196336

\section{HAL Id: halshs-00196336 \\ https://shs.hal.science/halshs-00196336}

Submitted on 12 Dec 2007

HAL is a multi-disciplinary open access archive for the deposit and dissemination of scientific research documents, whether they are published or not. The documents may come from teaching and research institutions in France or abroad, or from public or private research centers.
L'archive ouverte pluridisciplinaire HAL, est destinée au dépôt et à la diffusion de documents scientifiques de niveau recherche, publiés ou non, émanant des établissements d'enseignement et de recherche français ou étrangers, des laboratoires publics ou privés. 


\section{Au-delà de Kyoto : enjeux d'équité et d'efficacité dans la négociation sur le changement climatique}

\author{
Odile Blanchard $^{(*)}$ \\ Patrick Criqui $^{(*)}$ \\ Michel Trommetter $^{(* *)}$ \\ Laurent Viguier $^{(*)}$
}

(*) Institut d'Economie et de Politique de l'Energie (IEPE), Grenoble.

(**) Institut National de Recherche Agronomique (INRA), Grenoble.

Nous remercions N. Kouvaritakis (IPTS) pour sa contribution aux premières élaborations du scénario 2030, O. Godard pour ses remarques et commentaires et les deux rapporteurs.

Économie et Prévision

$n^{\circ} 143-1442000-2 / 3$
Les risques associés au changement climatique global pèsent sur l'ensemble de la communauté internationale. Mais dans le processus de discussion pour la maîtrise collective des émissions de gaz à effet de serre, et en particulier du gaz carbonique $\left(\mathrm{CO}_{2}\right)$ énergétique, tous les pays n'ont pas les mêmes intérêts, ne jouent pas le même rôle et ne proposent pas les mêmes objectifs.

La première difficulté à surmonter dans la négociation internationale a été et demeure celle de la répartition des efforts ou du «burden sharing », qui renvoie évidemment à la question de l'équité dans la détermination des objectifs. Ce fut, au moment de Kyoto, la question de la différenciation - ou non-différenciation - des objectifs quantitatifs des pays industrialisés, ce sera demain celle des règles à retenir pour l'identification de ceux des pays en développement. L'acceptabilité des objectifs est en effet une condition préliminaire de l'implication réelle des États dans le processus de discussion, comme dans les actions concrètes à entreprendre pour réduire les émissions.

Une base d'accord, concernant les pays industrialisés, s'est dessinée lors de la troisième «Conférence des Parties » à Kyoto, fin 1997. Mais une fois ces objectifs acceptés, se pose toujours aujourd'hui la question de la régulation des dispositifs de flexibilité. Étant donné la nature globale des impacts, il est en effet possible pour un pays d'obtenir les mêmes effets globaux, avec des coûts moindres, en profitant des potentiels de réduction à coût plus faible dans un autre pays. Les Parties à la Convention discutent et négocient actuellement les conditions de mise en œuvre de ces dispositifs de flexibilité, sujet qui sera notamment au cœur de la sixième « Conférence des Parties » à La Haye.

Cependant, les questions d'équité et d'efficacité deviennent encore plus aigües lorsque, dans une perspective «post-Kyoto » et en devançant à peine le calendrier des négociations internationales, on s'interroge sur les conditions de l'association des pays en développement à l'effort de réduction des émissions mondiales, sur la base d'objectifs quantitatifs (ou QELROs ${ }^{(1)}$ ).

Ce papier est fondé sur le constat que les intérêts divergents des pays ne peuvent pas mener à une solution qui repose sur une règle unique de différenciation applicable à tous. Il conviendrait alors plutôt d'examiner des solutions pragmatiques et d'évaluer ex post leur validité au regard de différentes conceptions de l'équité. C'est en effet parce qu'il n'existe pas de critère d'équité universel qu'il est nécessaire de mettre en œuvre des critères multiples pour apprécier l'acceptabilité internationale d'une solution donnée. Pour ce faire, on procédera en deux grandes parties, consacrées tour à tour aux questions d'équité, puis à 
l'élaboration d'un scénario mondial à 2030 impliquant les pays en développement. L'acceptabilité de ce scénario pour les différentes parties dans la négociation sera enfin examinée, à la lumière de plusieurs critères.

La première partie présente donc une revue des règles de différenciation des objectifs évoquées dans le processus de négociation internationale sur l'effet de serre. Elle se poursuit par le rappel des principales conceptions de l'équité, ainsi que de leurs fondements. Elle tente enfin de construire un lien entre les règles de différenciation exposées et les divers principes d'équité.

La deuxième partie propose la quantification d'un scénario d'attribution des droits d'émission à 2030 pour tous les pays du monde. Elle montre qu'à partir d'un programme de réduction conduisant à une stabilisation progressive des émissions après 2030, l'instauration d'un marché de permis d'émission permettrait de se rapprocher du programme efficace et donc de diminuer considérablement le coût total de l'action. Enfin, le scénario proposé est examiné à la lumière d'un ensemble de critères visant à le qualifier du point de vue de l'équité internationale et donc à apprécier les conditions de son acceptabilité.

\section{Quelle différenciation pour quelle équité internationale?}

L'objet de cette partie est l'identification des critères et des règles susceptibles de permettre à terme la participation des pays en développement à l'effort de limitation des émissions de gaz à effet de serre. Après avoir analysé les principales règles de différenciation qui ont été proposées par des pays ou exposées dans la littérature, nous tenterons de montrer, dans chaque cas, à quel(s) principe(s) d'équité elles font référence.

La différenciation des objectifs de réduction des émissions : règles, critères et enjeux

Après une première conférence des chefs d'État à La Haye en 1989, la prise de conscience de la communauté internationale vis-à-vis de l'effet de serre s'est ouvertement manifestée en 1992, lors du "Sommet de la Terre » à Rio de Janeiro. La Convention Cadre sur le Changement Climatique (UNFCCC, 1992) fut alors signée par la plupart des pays représentés. En décembre 1997, la troisième «Conférence des Parties » de la Convention aboutit à la rédaction du Protocole de Kyoto (UNFCCC, 1997) mais la signature de ce Protocole par les Parties n'est pas intervenue aussi rapidement ${ }^{(2)}$. Il est vrai que les engagements n'ont pas la même portée dans les deux textes. Dans la Convention, il s'agit d'accords généraux non contraignants légalement, à l'inverse du Protocole de Kyoto (encadré 1).

\section{Encadré 1 :Convention Cadre sur le Changement Climatique, Protocole de Kyoto : quelles avancées dans la lutte contre le changement climatique ?}

Dans la Convention Cadre, les Parties reconnaissent comme objectif ultime la stabilisation de la concentration dans l'atmosphère des gaz à effet de serre, à un niveau qui empêche toute perturbation anthropique dangereuse du système climatique. Toutes les Parties s'engagent à publier régulièrement des inventaires de leurs émissions de gaz à effet de serre, à mettre en place des mesures de prévention et d'adaptation au changement climatique. Par ailleurs, les Parties figurant à l'annexe 1 de la Convention (" pays développés et autres Parties développées », incluant les pays de l'OCDE, l'Union Européenne et les pays en transition vers une économie de marché) s'engagent à stabiliser leurs émissions de gaz à effet de serre au niveau de 1990 ; les pays et autres Parties développées figurant à l'annexe 2 (pays de l'OCDE + Union Européenne) s'engagent également à financer les coûts engagés par les pays en développement pour respecter leurs engagements.

Par rapport à la Convention, les principaux éléments nouveaux du Protocole de Kyoto comprennent :

- l'engagement des parties figurant à l'annexe B (Parties citées à l'annexe 1 de la Convention et ayant ratifié la
Convention) de réduire globalement leurs émissions d'au moins $5 \%$ entre 2008 et 2012 par rapport à 1990 ;

- le recours possible aux « mécanismes de flexibilité » que constituent le marché de permis d'émission, l'application conjointe et le mécanisme de développement propre ; le marché de permis d'émission est accessible aux seuls pays ayant accepté des engagements de réduction de leurs émissions ; de ce fait, on peut dire que le Protocole de Kyoto a établi la répartition initiale des permis d'émission entre les pays de l'annexe B ; l'application conjointe et le mécanisme de développement propre sont des mécanismes de flexibilité liés à des projets : la première vise, pour un pays donné de l'annexe $\mathrm{B}$, à obtenir des unités de réduction d'émissions en échange du financement de projets de réduction d'émissions dans un autre pays annexe $\mathrm{B}$; le second permet de délivrer, sur une base multilatérale, des certificats de réductions d'émissions à un pays annexe $\mathrm{B}$ donné, en échange de projets de réduction financés dans un pays en développement.

Les engagements des pays en développement dans le Protocole sont en revanche limités à ceux définis dans la Convention. 
En 1997, le Protocole de Kyoto fut perçu comme un succès, en tant que première étape indispensable dans le long processus de négociation sur l'effet de serre. Pourtant, outre l'incertitude actuelle sur le rythme de signature et de ratification, ce texte contient de nombreux dispositifs dont les modalités d'application restent à définir. Lors de la quatrième Conférence des Parties (COP 4) à Buenos Aires (novembre 1998), un Plan d'Action de deux ans a d'ailleurs été adopté : ainsi, fin 2000, à la sixième Conférence des Parties (COP 6) de La Haye, les modalités de fonctionnement des «mécanismes de flexibilité » et les règles de respect des obligations des Parties ayant ratifié le Protocole devraient-elles définitivement être mises en place ${ }^{(3)}$.

Quant à la question de l'association des pays en développement aux efforts de limitation des émissions, elle est récurrente à chaque Conférence des Parties et reste toujours ouverte avant celle de La Haye. Les émissions étant appelées à croître très fortement dans ces pays dans les prochaines décennies ${ }^{(4)}$, les efforts des régions plus industrialisées ne pourront en aucun cas suffire pour atteindre l'objectif de stabilisation des émissions puis de la concentration des gaz à effet de serre dans l'atmosphère. Par conséquent, la réflexion sur les façons d'associer les pays en développement à des engagements contraignants est inévitable et elle doit conduire à définir le " cour » du futur dispositif mondial de lutte contre l'effet de serre.

Évidemment, les pays en développement ne peuvent pas aujourd'hui accepter, comme les pays de l'annexe B, de réduire ou même de stabiliser leurs émissions à un horizon aussi proche que 2008-2012. Leurs consommations d'énergie et leurs émissions sont en forte croissance, du fait de la dynamique démographique et des changements structurels de leurs économies. Par conséquent, la différenciation des objectifs d'évolution des émissions ${ }^{(5)}$ entre les pays industrialisés et ceux des pays en développement s'avère indispensable. Il en est d'ailleurs de même au sein des pays en développement, car ceux-ci ne forment en aucune manière un groupe homogène.

\section{La différenciation, élément structurant de la Convention Cadre et du Protocole de Kyoto}

Introduit dans de nombreux articles de la Convention, le terme "différenciation » y est pris dans son acception large, qui est de tenir compte des spécificités nationales pour atteindre l'objectif ultime de la Convention. Cependant, nous utiliserons ici le terme «différenciation » dans le sens plus restrictif de "différenciation des objectifs quantitatifs de limitation et de réduction des émissions», dans la mesure où notre réflexion porte sur la façon dont ces objectifs de limitation peuvent être répartis entre les pays.
Retenir le principe de la différenciation permet de prendre en compte, au moins partiellement, les situations particulières des pays, du point de vue de leurs émissions et de leur participation à l'effet de serre. Dans la Convention, les objectifs de réduction des émissions sont différenciés selon les groupes de pays. D'un côté, les Parties de l'annexe 1 s'engagent à stabiliser leurs émissions de gaz à effet de serre au niveau de 1990 à l'horizon 2000. De l'autre, les Parties non-annexe 1 n'ont aucun engagement de ce type. La différenciation s'opère alors selon un axe « Nord-Sud », en fonction de la responsabilité différenciée des Parties dans la formation de l'effet de serre.

Dans le Protocole de Kyoto ensuite, à cette différenciation Nord-Sud vient s'ajouter une différenciation - que nous qualifierons de secondaire - à l'intérieur des Parties figurant à l'annexe B, pour atteindre l'objectif de réduction des émissions de gaz à effet de serre d'au moins $5 \%$ globalement entre 2008 et 2012 par rapport à 1990 . Un taux de réduction des émissions presque uniforme (flat rate) est alors adopté pour la triade Union Européenne (-8 \%), États-Unis (-7\%), Japon $(-6 \%)$. La différenciation est cependant plus marquée lorsqu'on examine les taux d'évolution des émissions sur lesquels se sont engagées l'Islande $(+10 \%)$, l'Australie $(+8 \%)$, la Norvège $(+1 \%)$, la Nouvelle-Zélande, la Fédération de Russie et l'Ukraine $(+0 \%)$.

Sur quelles bases et selon quels principes la différenciation pourrait-elle être mise en œuvre pour les pays en développement ? De nombreuses propositions ont été formulées dans la littérature scientifique ou par certains pays, depuis la fin des années quatre-vingts et surtout avant les négociations de Kyoto.

\section{La multiplicité des règles de différenciation}

La plupart des pays retiennent non pas une règle élémentaire mais un schéma de différenciation combinant plusieurs règles élémentaires. Sur ces bases, certains pays se sont attachés à définir clairement les modalités de calcul de la différenciation ${ }^{(6)}$, tandis que d'autres ont simplement établi une liste des variables à prendre en compte (FCCC, 1996). Il en est de même dans la littérature sur le sujet : les règles sont présentées soit individuellement, soit en combinaison. Mais les modes opératoires d'application de ces règles sont bien souvent mal définis. Ceci atteste la difficulté potentielle à mettre en œuvre concrètement certaines des règles de différenciation ${ }^{(7)}$.

Il est cependant possible d'effectuer un classement de ces règles, selon qu'elles portent sur des volumes de réduction d'émissions, sur des coûts de réduction des émissions (le " partage de la charge ", burden sharing $)^{(8)}$, ou sur des indicateurs relatifs aux circonstances nationales. 
Pour la clarté de l'exposé, la distinction entre les deux premières catégories de différenciation (volumes de réduction, coûts de réduction) est utile. Mais elle est en partie artificielle, dans la mesure où à un objectif donné de réduction d'émissions correspond de fait un niveau déterminé de coût de réduction et inversement. En tout cas, les propositions des pays concernent dans leur majorité une différenciation basée sur des volumes de réduction d'émissions : cela s'explique évidemment par la plus grande difficulté à observer et à évaluer les coûts de réduction que les volumes d'émissions. En effet, ces derniers se déduisent aisément des consommations d'énergie, alors que la détermination des coûts de réduction nécessite la prise en compte d'un grand nombre de variables et généralement l'utilisation d'un modèle économique ou énergétique.

Les indicateurs relatifs aux circonstances nationales correspondent, quant à eux, aux facteurs qu'il est possible de prendre en compte pour la différenciation ; ils reflètent très souvent les intérêts des pays qui les proposent. Ils sont généralement combinés entre eux et/ou avec des règles de différenciation de l'une des deux premières catégories. Ils sont cependant peu susceptibles d'être retenus dans le cadre de la négociation internationale, puisqu'ils ont peu de chances de recueillir le consensus d'un nombre suffisant de pays $^{(9)}$.

Par la suite, nous nous intéressons donc seulement aux deux grandes catégories de différenciation, portant sur les volumes ou sur les coûts. Pour chacune, le tableau 1 expose les principales règles retenues et les critères qui leur sont associés.
Dans les deux catégories, certaines règles sont généralement présentées comme étant plutôt favorables aux pays en développement, d'autres aux pays industrialisés. En particulier :

- si à terme un plafond d'émissions par habitant était fixé, sa valeur serait inévitablement inférieure à celle déjà atteinte par les pays industrialisés et supérieure à celle des autres pays ; les pays en développement pourraient ainsi encore laisser croître leurs émissions par habitant pendant une certaine période $^{(10)}$;

- baser la différenciation sur le PIB par habitant permettrait de tenir compte, pour les pays en développement, de la priorité donnée à la satisfaction des besoins fondamentaux et, pour les pays industrialisés, de leur capacité à payer ; en revanche, cette règle n'étant pas liée directement aux émissions ou à leurs coûts de réduction, elle peut dans certains cas ne pas être suffisamment incitative; - une différenciation définie en fonction de la contribution des pays au changement climatique mènerait à ce que les volumes de réduction ou les coûts totaux de réduction soient proportionnellement plus faibles dans les pays en développement que dans les pays industrialisés, si la mesure de la contribution s'effectuait au regard des seules émissions passées ${ }^{(11)}$;

- la répartition des quotas d'émissions au prorata des émissions actuelles ou cumulées (quotas « hérités » ou grandfathering) favoriserait les pays industrialisés puisque les émissions actuelles constitueraient un "droit acquis » (Godard, 1997), mais elle pénaliserait fortement les pays en développement qui ne disposeraient pas alors de marge de manœuvre pour augmenter leurs émissions dans les prochaines décennies ;

- si l'effort de réduction des émissions (en volume ou en coût) était déterminé en fonction de l'intensité

\section{Tableau 1 : principales règles de différenciation et critères correspondants}

\begin{tabular}{|c|c|}
\hline $\begin{array}{l}\text { Règles de différenciation basées sur les volumes de réduction } \\
\text { des émissions }\end{array}$ & Critères de différenciation \\
\hline Plafond d'émissions par habitant à ne pas dépasser & Emissions par habitant \\
\hline $\begin{array}{l}\text { Objectif de réduction proportionnel au produit intérieur brut }(P I B) \text { par } \\
\text { habitant }\end{array}$ & $P I B$ par habitant \\
\hline $\begin{array}{l}\text { Volume de réduction proportionnel à la contribution du pays aux } \\
\text { émissions mondiales ou au réchauffement global }\end{array}$ & Emissions actuelles ou cumulées, part dans le réchauffement global \\
\hline $\begin{array}{l}\text { Quota d'émissions attribué sur la base des émissions actuelles ou } \\
\text { cumulées de chaque pays (grandfathering) }\end{array}$ & Emissions actuelles ou cumulées \\
\hline Volume de réduction proportionnel aux émissions par unité de $P I B$ & Emissions / PIB \\
\hline $\begin{array}{l}\begin{array}{c}\text { Règles de différenciation basées sur les coûts de réduction } \\
\text { des émissions }\end{array} \\
\end{array}$ & Critères de différenciation \\
\hline $\begin{array}{l}\text { Coût total de réduction proportionnel au } P I B \text {, ou coût par unité de } P I B \\
\text { proportionnel au } P I B \text { par habitant }\end{array}$ & $P I B, P I B$ par habitant \\
\hline $\begin{array}{l}\text { Coût total de réduction proportionnel à la contribution du pays aux } \\
\text { émissions mondiales ou au réchauffement global }\end{array}$ & Emissions actuelles ou cumulées, part dans le réchauffement global \\
\hline Coût de réduction proportionnel aux émissions par unité de $P I B$ & Emissions / PIB \\
\hline Egalisation des coûts marginaux de réduction & Coût marginal de réduction \\
\hline Coût total supporté en fonction du consentement à payer & Utilité de l'atténuation du changement climatique \\
\hline
\end{tabular}


d'émission $d u$ PIB, les pays dont les systèmes énergétiques seraient relativement plus émetteurs de $\mathrm{CO}_{2}$ devraient effectuer les efforts les plus importants; cette règle de différenciation est perçue comme défavorable aux pays, industrialisés ou en développement, dont les systèmes énergétiques sont fortement émetteurs de gaz à effet de serre (États-Unis, Inde, Chine par exemple) ;

- l'égalisation des coûts marginaux de réduction est une règle parfois présentée comme susceptible de conduire à des objectifs de réduction relativement élevés pour les pays ayant des systèmes énergétiques peu efficaces et présentant des potentiels de réduction à bas coût plus importants que ceux des pays plus efficaces; ce diagnostic devrait cependant être nuancé, en tenant compte au cas par cas de la structure du système énergétique et de la dynamique des besoins ; en outre, cette solution supposée optimale peut être mise en œuvre par l'application d'une taxe, mais plus difficilement à partir d'une approche sur les quantités ;

- si le coût de réduction dépendait du «consentement à payer ", il serait lié à l'utilité que chaque pays peut tirer de l'atténuation du changement climatique ; comme il est probable que les impacts négatifs les plus forts affecteront les pays en développement, les avantages de la prévention seraient a priori plus grands pour ces pays ; les coûts d'atténuation les plus importants devraient alors, dans cette optique, être consentis par les pays en développement ; mais ce diagnostic devrait aussi à l'évidence être considérablement amendé par la prise en compte des moyens financiers très limités dont ces pays disposent pour satisfaire leurs priorités de développement.

Les règles présentées dans le tableau 1 montrent bien les divergences d'intérêt. Mais, même en appliquant une règle globalement favorable aux pays en développement, certains pays du groupe seraient plus avantagés que d'autres, en fonction de leurs caractéristiques propres et de la règle de différenciation précise qui serait retenue. L'analyse que nous venons de mener montre l'importance des enjeux associés à ce type de choix. Comme l'indique Müller (1998), la probabilité est faible pour qu'une règle unique apparaisse équitable à tous les pays : seules des solutions " mixtes » permettront de recueillir un consensus suffisant et pourront être retenues. Il faut alors remonter de l'analyse des règles et des critères à celle des principes susceptibles de fonder des approches acceptables en termes d'équité internationale.

\section{Des règles de différenciation aux principes de justice}

Il s'agit donc dans cette partie d'analyser les fondements éthiques des différentes règles de différenciation des émissions de $\mathrm{CO}_{2}$. Cette analyse mériterait une réflexion sur les problèmes que pose l'application des principes de justice aux relations internationales ${ }^{(12)}$ et à un bien tel que les émissions $\mathrm{de} \mathrm{CO}_{2}{ }^{(13)}$. Nous nous contenterons de déterminer sur quels principes de justice reposent les propositions formulées pour répartir les droits d'émission de $\mathrm{CO}_{2}$ à l'échelon mondial.

Six conceptions principales de la justice sont tour à tour envisagées : l'égalité des droits, l'égalité utilitariste, l'égalité démocratique, la responsabilité causale, le mérite et l'égalité proportionnelle. L'antinomie entre ces différentes conceptions de la justice n'est pas totale : si par exemple le mérite et l'égalité proportionnelle contredisent tous deux le principe d'égalité des droits, l'égalité démocratique se fonde en partie sur l'égalité des droits et la responsabilité causale est compatible avec l'utilitarisme.

\section{L'égalité des droits}

Tel que défini par la théorie du droit naturel (Locke, 1690), le principe d'égalité des droits peut servir de référence pour distribuer les droits d'émission à l'échelon international. Selon cette approche, les hommes sont considérés comme naturellement dans un état de parfaite liberté et d'égalité, c'est-à-dire un état où tout pouvoir et toute juridiction sont réciproques : nés sans distinction, ayant part aux même avantages de la nature et ayant les mêmes facultés, les hommes doivent nécessairement être égaux entre eux.

Dans le contexte du changement climatique, l'application stricte du principe d'égalité conduirait directement à accorder les mêmes droits à chaque individu en matière d'utilisation de l'atmosphère. Selon ce principe égalitaire, les droits d'émission seraient distribués directement aux individus, et non aux gouvernements, et chacun se verrait accorder exactement la même quantité de gaz à effet de serre.

\section{L'égalité utilitariste}

L'égalité utilitariste est une conception de la justice distincte de l'égalité des droits. L'utilitarisme enseigne qu'une action ne peut être jugée moralement bonne ou mauvaise qu'en mesure de ses conséquences pour le bonheur des individus concernés (Audard, 1996) : le plus grand bonheur pour le plus grand nombre est la seule fin universellement désirable (Bentham, 1789). L'objectif utilitariste est de maximiser l'utilité non pas individuelle mais totale, calculée comme un solde net des satisfactions par rapport aux désavantages, chaque individu comptant également et étant traité de manière impartiale. On remarque que l'utilitarisme ignore les questions de répartition: le gain le plus infime de la somme totale des utilités l'emporte par conséquent sur les inégalités distributives les plus criantes (Sen, 1993).

$\mathrm{Au}$ regard de l'utilitarisme, la solution juste en matière de réduction des émissions de $\mathrm{CO}_{2}$ à l'échelle internationale serait celle qui maximiserait la croissance économique mondiale, nette des 
dommages climatiques, même si ces dommages sont concentrés sur certaines régions « sacrifiées ». Dans cette optique, les États auraient la liberté de décider des réductions qu'ils consentent à payer (« willingness to pay »), selon leurs préférences individuelles. Les pays en développement, probablement les plus vulnérables aux changements climatiques, devraient ainsi optimiser leur développement en investissant dans des mesures de réduction des émissions seulement si celles-ci sont susceptibles de contribuer davantage à leur développement futur que les investissements directs réalisés pour le développement (Schelling, 1992). Les gagnants nets de la prévention (ceux qui sont surtout victimes de la pollution) pourraient aussi être conduits à proposer des compensations attractives aux perdants nets de la prévention (ceux qui sont surtout pollueurs) pour qu'ils limitent leur pollution (Godard, 1997).

Toutefois, les avantages de la prévention sont difficiles à évaluer pour les différents pays et l'analyse coûts-avantages qui s'impose en théorie doit céder la pas à une analyse en termes de coûts-efficacité, où l'objectif global de réduction des émissions est fixé ex ante. En vertu de l'égalité utilitariste, la répartition de l'effort pour atteindre l'objectif global consisterait alors à déterminer pour chaque pays l'objectif de réduction qui permette d'égaliser les coûts marginaux de réduction entre les pays (et donc de minimiser le coût total de réduction). Cela conduirait à concentrer l'effort de réduction des émissions dans les pays ou les secteurs offrant les plus importants gisements de réduction à bas coût, sans que ceux-ci soient nécessairement les plus riches ou les plus gros émetteurs de $\mathrm{CO}_{2}$. Cette solution pourrait être réalisée par le biais des instruments dits " économiques 》 de la politique environnementale (taxe et/ou permis négociables).

\section{L'égalité démocratique}

L'égalité démocratique est la conception de la justice définie par John Rawls (1971). Ce dernier a en effet entrepris d'établir les principes de justice qui devraient gouverner la « structure de base » d'une société juste. Selon lui, placés dans une situation de parfaite impartialité (« le voile d'ignorance »), les individus devraient se mettre d'accord sur les deux principes de justice suivants. Premier principe : chaque personne doit avoir un droit égal au système le plus étendu de libertés de base qui soit compatible avec le même système pour les autres. Second principe : les inégalités sociales et économiques doivent être organisées de façon à ce que, à la fois, elles apportent aux plus désavantagés les meilleures perspectives et qu'elles soient attachées à des fonctions et à des positions ouvertes à tous, conformément à la juste (fair) égalité des chances. Le second principe contient le « principe de différence » donnant la priorité à la défense des intérêts des plus défavorisés et affirmant qu'il suffit que la position du plus mal loti s'améliore pour que l'état final soit considéré comme plus juste que l'état initial. Ce critère de classement est nommé " maximin » puisqu'il consiste à maximiser l'accroissement des «biens premiers » de ceux qui occupent la position minimale.

Appliquer le principe d'égalité démocratique à la lutte contre l'effet de serre revient à considérer que les ressources de l'atmosphère sont des « biens premiers ». Si on suppose qu'émettre des gaz à effet de serre fait partie des attributs fondamentaux de la personne, une répartition juste des droits d'émission, selon le principe de différence, serait celle qui maximiserait les bénéfices nets des pays les plus pauvres. Les règles de différenciation devraient alors tenir compte de la capacité des États à payer et permettre une répartition de la charge qui soit favorable aux pays les plus défavorisés. Des règles de différenciation proposées, celles qui envisagent de distribuer une plus large proportion des quotas d'émissions aux pays les plus pauvres ou de faire supporter des coûts plus importants aux pays les plus riches, répondent à cette conception « rawlsienne » de l'équité.

\section{La responsabilité causale}

Le principe de responsabilité causale découle, dans la théorie utilitariste et plus précisément dans l' «économie du bien-être », du souci de prise en compte des effets externes dans l'économie. Les effets externes regroupent un ensemble de phénomènes que Pigou décrit comme des «services non compensés »- résultant d'une divergence entre le produit marginal social net et le produit marginal privé net - à l'origine d'une allocation sous-optimale des ressources dans la société (Pigou, 1932). La solution pigouvienne au problème des externalités, l'internalisation, revient à évaluer monétairement les coûts et avantages procurés sans compensation pécuniaire. Il s' agit d'établir le prix de l'effet externe qui égalise le produit marginal social et le produit marginal privé. Quand l'externalité est négative, l'internalisation peut prendre la forme d'un impôt (taxe pigouvienne) ou d'une norme réglementaire. On parle alors, dans le cas de la pollution, de principe pollueur-payeur, puisqu'il s'agit d'imputer la responsabilité de l'effet externe à celui qui en est à l'origine.

Dans le cadre de la lutte contre le réchauffement climatique, l'application du principe de responsabilité causale consisterait à appliquer au niveau international le principe pollueur-payeur. Chaque pollueur serait tenu de supporter le coût des dépenses de réduction de sa propre pollution. Cette solution pourrait être réalisée par le biais des instruments économiques (taxe et/ou permis négociables) ou par l'application d'une norme sur les émissions de $\mathrm{CO}_{2}$. Dans le cas où l'on opterait pour un système de taxation, il faudrait choisir entre la création d'une taxe uniforme sur les émissions actuelles de $\mathrm{CO}_{2}$ (taxe incitative) ou une répartition 
du coût de certaines actions de réduction proportionnelle aux émissions actuelles (taxe de financement). Mais il faudrait auparavant s'entendre sur la nature et l'étendue de la responsabilité (émissions actuelles ou émissions cumulées?).

\section{Le mérite}

Le mérite est le fondement de la conception de la justice retenue par Marx (1875) pour la première étape du communisme. Marx préconisait de donner « à chacun selon son travail ». Les dotations de chacun sont considérées comme une récompense de la bonne conduite - la recherche de la vertu, les efforts fournis, etc. - et sont donc réparties en fonction des mérites correspondant à des comportements (Van Parijs, 1991). Les thèses méritocratiques admettent par conséquent les inégalités distributives qui naissent de l'effort, de la compétence, du talent, du risque, de la responsabilité ou du courage (Dupuy, 1992).

L'application de ce principe à la lutte contre le réchauffement climatique pourrait conduire à tenir compte des performances environnementales des différents pays dans la gestion des réductions d'émissions, considérant qu'il y a un " mérite » à posséder une économie relativement peu polluante. Une répartition des droits d'émission tenant compte des niveaux d'intensité d'émission (les émissions par unité de $P I B$ ) pourrait s'accorder avec un tel principe, si elle conduisait à pénaliser les économies intensives en $\mathrm{CO}_{2}$ et à récompenser celles qui ne le sont pas.

\section{L'égalité proportionnelle}

L'égalité proportionnelle est la conception de la justice telle que définie par Aristote. Il convient, selon Aristote, qu'à des personnes inégales reviennent des choses inégales. Dans la philosophie scolastique prévalait encore cette conception de la justice distributive qui implique que soit rendu à chacun ce qui lui est dû, selon son rang. La position sociale est le facteur structurant de la répartition et elle est considérée comme le seul critère effectif de comparaison entre les individus, car la fonction qu'un individu occupe dans la société est supposée correspondre à la valeur de son travail pour la société. Les théories libertariennes de Nozick (1996) et la théorie du «marchandage rationnel» de Gauthier (1986) répondent à cette conception dite « historique » de la justice. Selon ces auteurs, justice est faite lorsque les avantages dont la distribution est importante sont conçus comme des droits acquis par un achat, un don, un legs, un effort coopératif ou une découverte (Van Parijs, 1998).

Selon cette conception de la justice, les émissions existantes et/ou passées pourraient représenter un «droit acquis » des États. Une répartition conforme à ce principe reviendrait par conséquent à répartir les quotas d'émissions futures au prorata de ces droits acquis et à reproduire ainsi les inégalités en matière d'utilisation de l'atmosphère (droits « hérités » ou " grandfathering »). Peu d'auteurs ont perçu cette relation entre les conceptions « historiques » de la justice et les règles d'allocation des droits d'émission basées sur le «statu quo » ${ }^{(14)}$. À défaut de trouver un fondement éthique au critère des émissions passées, la plupart des auteurs se réfèrent au droit coutumier (Young et Wolf, 1991). Difficilement justifiable d'un point de vue éthique, la règle du statu quo se présente comme une allocation «par défaut » qui, en l'absence d'accord entre les parties, n'impose pas automatiquement un très large effort de la part des gros pollueurs (Grubb, 1995).

Le tableau 2 constitue une synthèse des principes d'équité évoqués dans la négociation. Parmi l'ensemble des règles de différenciation proposées (tableau 1), existe-t-il une règle renvoyant à un principe d'équité (tableau 2) susceptible de constituer une norme juste aux yeux de tous? Sans doute pas, car aucun principe libéral de justice distributive, y compris le principe de différence, ne

Tableau 2 : application des principes d'équité dans la lutte contre l'effet de serre

\begin{tabular}{|c|c|}
\hline Règles de limitation des émissions de $\mathrm{CO}_{2}$ & Principes de justice \\
\hline Egalisation des émissions par habitant & L'égalité des droits \\
\hline $\begin{array}{l}\text { Coût total supporté en fonction du consentement à payer } \\
\text { Egalisation des coûts marginaux de réduction }\end{array}$ & L'égalité utilitariste \\
\hline $\begin{array}{l}\text { Objectif de réduction des émissions proportionnel au } P I B \text { par habitant } \\
\text { Coût total de réduction proportionnel au } P I B \text { ou coût par unité de } P I B \text { proportionnel } \\
\text { au } P I B \text { par habitant }\end{array}$ & L'égalité démocratique \\
\hline $\begin{array}{l}\text { Volume de réduction proportionnel à la contribution du pays aux émissions } \\
\text { Coût total de réduction proportionnel à la contribution du pays aux émissions }\end{array}$ & La responsabilité causale \\
\hline $\begin{array}{l}\text { Volume de réduction des émissions proportionnel aux émissions par unité de } P I B \\
\text { Coût total de réduction des émissions proportionnel aux émissions par unité de } P I B\end{array}$ & Le mérite \\
\hline $\begin{array}{l}\text { Quota d'émissions attribué sur la base des émissions actuelles ou cumulées de } \\
\text { chaque pays (grandfathering) }\end{array}$ & L'égalité proportionnelle \\
\hline
\end{tabular}


semble pouvoir être jugé acceptable par toutes les sociétés - mêmes libérales - pour résoudre la question du « partage du fardeau ». Le problème théorique est en effet celui de l'application des principes de justice, élaborés pour régir les inégalités au sein d'une société, aux relations internationales : on ne peut espérer que l'ensemble des États réponde à une conception commune de la justice distributive (Rawls, 1996). Comme le souligne James Fishkin (1986), «l'éthique des relations internationales, plus clairement que celle de la justice intérieure, dévoile la nécessité du conflit moral, de l'équilibre entre considérations incommensurables, et donc l'impossibilité de satisfaire des attentes absolutistes ».

Ce «conflit moral» existe en matière de lutte internationale sur le changement climatique. Le Protocole de Kyoto en témoigne : la différenciation des objectifs de réduction des émissions des pays de l'annexe B, retenue à Kyoto, ne repose pas sur une conception particulière de la justice. Elle apparaît plutôt comme un compromis négocié entre des pays divergents à la fois dans leurs valeurs, dans leurs intérêts et dans les anticipations qu'ils peuvent former des coûts de l'action (Viguier, 1999). Ce constat devrait conduire à adopter une démarche pragmatique pour aborder la question de l'intégration des pays en développement dans le processus de réduction des émissions au-delà de 2010. À défaut de pouvoir s'entendre sur une distribution des droits d'émission à l'échelle de la planète qui réponde à un principe de justice, il convient de proposer une solution qui permette de maximiser la participation des États à un coût « acceptable » en tenant compte :

- de l'intérêt collectif, par exemple d'un objectif de stabilisation des émissions mondiales de $\mathrm{CO}_{2}$ à l'horizon 2030, dans le respect des considérations d'efficacité économique ;

- de la responsabilité des pays développés quant au phénomène de réchauffement climatique, avec en particulier la poursuite, au delà de 2010, de l'effort de réduction déjà engagé pour cet horizon ;

- des intérêts des pays en développement, par la prise en compte des contraintes et des dynamiques démographiques, économiques et énergétiques auxquelles on doit s'attendre dans ces pays.

Une telle démarche ne repose pas sur l'application ex ante d'un principe de justice, mais elle est construite dans l'optique d'une validation ex post du scénario proposé s'appuyant sur un ensemble d'indicateurs ou critères pertinents au regard de différentes conceptions de la justice (telles qu'en particulier l'égalité des droits ou l'égalité démocratique).

\section{Un scénario de stabilisation des émissions mondiales en 2030}

Certaines des règles envisagées pour la différenciation des objectifs de réduction ont pour avantage de ne nécessiter ni l'identification d'une projection de référence, ni une évaluation des coûts de réduction. C'est le cas notamment des objectifs déterminés en termes d'émissions par tête ou en taux de réduction par rapport à une année de base. Cette dernière solution a pu être jugée acceptable par les pays de l'annexe 1 dans le processus de Kyoto, avant tout parce qu'elle s'appliquait, soit à des pays industrialisés, dans lesquels les émissions ne devraient connaître qu'une augmentation modérée, soit aux pays en transition, dans lesquels les émissions ont même beaucoup décru au cours des dernières années. L'adoption par les pays industrialisés d'objectifs clairs et contraignants a constitué alors une avancée majeure dans le processus de la négociation internationale.

Cette règle n'a pu être étendue aux pays en développement, tout d'abord car ceux-ci refusent encore de s'engager sur des objectifs d'émissions contraignants. Il est d'autre part évident que la règle d'un plafond d'émissions construit en référence à une année passée ne peut leur être appliquée : dans la plupart de ces pays en effet, les tendances en cours de la démographie et de l'économie devraient entraîner une multiplication des émissions par un facteur deux ou trois entre 1990 et 2010.

La conférence de Kyoto a donc débouché sur une première attribution des droits ou contraintes d'émission de $\mathrm{CO}_{2}$ au plan mondial. Le principal défi est aujourd'hui de concevoir des scénarios «post-Kyoto » permettant d'analyser les conditions d'insertion des pays en développement dans un dispositif d'objectifs de réduction des émissions par rapport à la référence au-delà de 2010.

Dans cette deuxième partie, nous tenterons tout d'abord de construire un tel scénario, dans le cadre d'une approche pragmatique, tenant compte des contraintes imposées par le système climatique et des dynamiques économiques et énergétiques régionales. Il convient aussi de souligner que la démarche de construction du scénario s'inspirera fortement du processus suivi à Kyoto différenciation par groupe de pays, variables considérées, mode de formulation des objectifs pour tenter de l'adapter à la situation des pays en développement.

Dans un deuxième temps, nous fournirons les enseignements préliminaires de l'analyse quantitative de ce scénario en termes de coûts marginaux de réduction nationaux, puis de «valeur internationale du carbone» dans un système de 
marchés de droits d'émission. Nous montrerons en particulier que l'instauration d'un marché de permis d'émissions permet ex post de rétablir l'efficacité qui n'existe pas a priori dans la répartition initiale des droits. Enfin, nous évaluerons le scénario proposé en termes d'équité et envisagerons ses conditions d'acceptabilité par les différentes Parties à la négociation.

\section{Un scénario pragmatique pour un processus de stabilisation des émissions en 2030}

Le scénario présenté est un scénario préliminaire, qui devra être encore affiné par la suite. Il a le mérite d'envisager, de façon pragmatique, l'intégration des pays en développement dans le processus de réduction des émissions au-delà de 2010. En effet, il considère en premier lieu les contraintes visant à limiter le changement climatique, tout en prenant en compte les contraintes économiques, énergétiques et démographiques des pays en développement.

\section{Les contraintes climatiques}

Certes, les incertitudes sur le changement climatique lié à l'augmentation de la concentration des gaz à effet de serre dans l'atmosphère restent nombreuses. Cependant, les Parties signataires de la ConventionCadre sur le changement climatique ont adopté dans ce domaine le principe de précaution, en se fixant pour objectif ultime de stabiliser les concentrations de gaz à effet de serre dans l'atmosphère à un niveau qui empêche toute «perturbation anthropique dangereuse du système climatique » (article 2 de la Convention : UNFCCC, 1992).

De son côté, le GIEC (Groupe d'Experts Intergouvernemental sur l'Evolution du Climat) a construit diverses trajectoires possibles d'évolution des émissions de $\mathrm{CO}_{2}$, à partir de 1990, pour parvenir à une stabilisation de la concentration de $\mathrm{CO}_{2}$ dans l'atmosphère à un niveau préalablement fixé. Quel que soit le niveau de concentration retenu $(350,450$, 550,750 ou 1000 ppmv $\left.^{(15)}\right)$ et compte tenu du temps de résidence du $\mathrm{CO}_{2}$ dans l'atmosphère, les trajectoires d'émissions suivent une phase de croissance, se stabilisent puis s'infléchissent plus ou moins fortement et plus ou moins rapidement (IPCC, 1996).

Les scénarios de stabilisation de la concentration à 550 ppmv sont fréquemment pris comme référence dans les études relatives aux réductions des émissions ${ }^{(16)}$, car ce sont des scénarios qui tiennent compte du principe de précaution et proposent des trajectoires d'émissions qui sont a priori réalistes. Le scénario d'attribution des droits d'émissions à 2030 que nous présentons ici est construit à partir de cette hypothèse de stabilisation de la concentration de $\mathrm{CO}_{2}$ à hauteur de 550 ppmv.
Pour atteindre la stabilisation à 550 ppmv à l'horizon 2150, le GIEC met en évidence une trajectoire qui serait à son maximum de 12 milliards de tonnes de carbone (GtC) autour de 2030 et qui serait décroissante ensuite. Si l'on soustrait environ $2 \mathrm{GtC}$ émises par l'agriculture, le système énergétique devrait émettre au plus $10 \mathrm{GtC}$ en 2030. Notre scénario repose donc principalement sur cette contrainte de stabilisation des émissions globales du système énergétique à hauteur de $10 \mathrm{GtC}$ peu après 2030. D'où sa définition en termes de stabilisation mondiale des émissions de $\mathrm{CO}_{2}$ à $10 \mathrm{GtC}$ en 2030.

Ensuite, nous avons reconduit la différenciation primaire introduite dans le Protocole de Kyoto, entre pays industrialisés et pays en développement, ceci afin de tenir compte de dynamiques énergétiques et économiques extrêmement différenciées :

- pour les pays de l'annexe $\mathrm{B}$, le taux de réduction des émissions entre 2010 et 2030 est le même que celui pour lequel les pays se sont engagés entre 1990 et 2010 dans le Protocole de Kyoto ; par exemple, l'objectif de réduction pour les États-Unis en 2030 est de $7 \%$ par rapport à 2010 et leurs émissions en 2030 ne devront donc pas dépasser $0,93 * 0,93$ fois leur niveau d'émissions de 1990 ; dans cette hypothèse «Kyoto puissance deux», la différenciation secondaire introduite dans le Protocole de Kyoto est donc renouvelée à l'identique ;

- pour les pays non-annexe B, la contrainte de stabilisation des émissions globales autour de 2030 impose que, à cette date, l'augmentation de leurs émissions soit au plus égale à la diminution des émissions des pays de l'annexe B. Par ailleurs, comme les émissions mondiales devront à terme diminuer pour parvenir à une stabilisation de la concentration, les émissions des pays non-annexe $\mathrm{B}$ devront à plus long terme se stabiliser puis diminuer.

\section{La prise en compte des contraintes des pays en développement}

Pour les pays non-annexe B, la démarche proposée consiste non pas à statuer a priori sur un niveau de stabilisation (absolu ou par tête) mais à fixer :

- un point de départ pour la détermination de leurs droits, c'est la situation de référence en 2010 ;

- des horizons de stabilisation de leurs émissions, différenciés selon les niveaux de $P I B$ par habitant et d'émissions par habitant en 2010 ;

- un taux de croissance initial de leurs émissions en 2010, différencié pour tenir compte des dynamiques démographiques ; c'est la décélération progressive de ce taux jusqu'aux dates de stabilisation par pays qui va conduire à la stabilisation globale, compte tenu des réductions attendues dans les pays annexe $\mathrm{B}$. 
Pour les pays non-annexe B, catégorie découlant de la différenciation primaire du Protocole de Kyoto, l'exercice consiste donc à construire tout d'abord un schéma de différenciation secondaire prenant en compte le niveau de revenu par habitant en 2000 et le niveau d'émissions par habitant, également en 2000. À ce schéma vient s'ajouter ensuite un niveau tertiaire de différenciation, tenant compte de la croissance démographique attendue entre 2000 et 2010.

La figure 1 met en évidence quatre catégories de pays, parmi lesquelles la première correspond globalement aux pays de l'annexe B et les suivantes distinguent les pays non- annexe $\mathrm{B}$, selon qu'ils font partie de la catégorie 2 (revenu et émissions élevés), 3 (revenu et émissions intermédiaires) ou 4 (revenu et émission faibles). Elle permet de justifier la différenciation primaire (pays annexe $\mathrm{B}$ / pays nonannexe B) et d'opérer la différenciation secondaire des horizons de stabilisation des émissions des pays non-annexe B.

Le principe retenu est que, pour tenir compte à la fois de la capacité des États à payer et de leur responsabilité causale, plus les niveaux de revenu et d'émissions sont élevés et plus l'horizon de stabilisation de leurs émissions doit être rapproché. Inversement, un pays pauvre à faibles émissions par tête ne devra stabiliser ses émissions que dans un futur plus lointain. Nous prenons ainsi pour hypothèse que les pays de la catégorie 2 devront stabiliser leurs émissions à partir de 2015 , ceux de la catégorie 3 à partir de 2030 et ceux de la catégorie 4 à partir de 2045, ce qui revient à intervenir sur le taux de croissance annuel autorisé des émissions de $\mathrm{CO}_{2}$. La liste des pays et régions figurant dans chaque catégorie se trouve en annexe.

Il reste alors à déterminer les profils intertemporels des objectifs de limitation des émissions des pays non-annexe $\mathrm{B}$, conduisant de la situation non contrainte en 2010 à la stabilisation des émissions mondiales à partir de 2030. Le taux de croissance initial en 2010 des émissions est fixé, selon l'hypothèse d'une croissance de $3 \%$ par an des émissions par habitant, uniforme pour tous les pays en développement ${ }^{(17)}$. Cela conduit à une différenciation tertiaire, par la prise en compte du taux de croissance démographique moyen annuel enregistré dans chaque pays entre 2000 et 2010. Le taux de croissance des émissions est donc initialement égal à $3 \%$ par an plus le taux de croissance démographique, puis il diminue linéairement chaque année pour atteindre la valeur zéro à la date de stabilisation $(2015,2030,2045)$.

En résumé, le scénario « Stabilisation mondiale à 10 $\mathrm{GtC}$ en 2030 » que nous proposons s'articule autour des points suivants pour l'attribution des droits d'émission : stabilisation globale des émissions en 2030, poursuite des réductions engagées dans les pays de l'annexe $\mathrm{B}$, ralentissement progressif mais différencié de la croissance des émissions des pays non-annexe B, la différenciation au sein de ces pays s'effectuant en fonction des niveaux de revenu et d'émissions par habitant mais aussi de la dynamique démographique. La formalisation mathématique de ce scénario figure dans l'encadré 2.

La figure 2 présente le résultat du scénario en termes d'émissions. Elle fait bien apparaître la stabilisation des émissions globales à $10 \mathrm{GtC}$ un peu après 2030 , la décroissance des émissions des pays annexe $\mathrm{B}$ sur toute la période, la stabilisation des émissions dès 2015 pour les pays de la catégorie 2 , en 2030 pour les pays de la catégorie 3 et en 2045 pour les pays de la catégorie 4.

\section{Évaluation économique du scénario : les gains de la flexibilité}

De nombreux travaux ont déjà montré l'intérêt économique de la mise en place d'un marché de permis d'émissions (Criqui, 1999 ; Energy Journal Special issue, 1999). Cette section vise à montrer les enjeux quantitatifs de l'instauration de mécanismes
Figure 1 : la différenciation des horizons de stabilisation
Les significations des codes des pays (ou régions) figurent en annexe.

Le $P I B /$ hab est exprimé en dollars constants de 1990, en parités de pouvoir d'achat.

Source : base de données modèle POLES

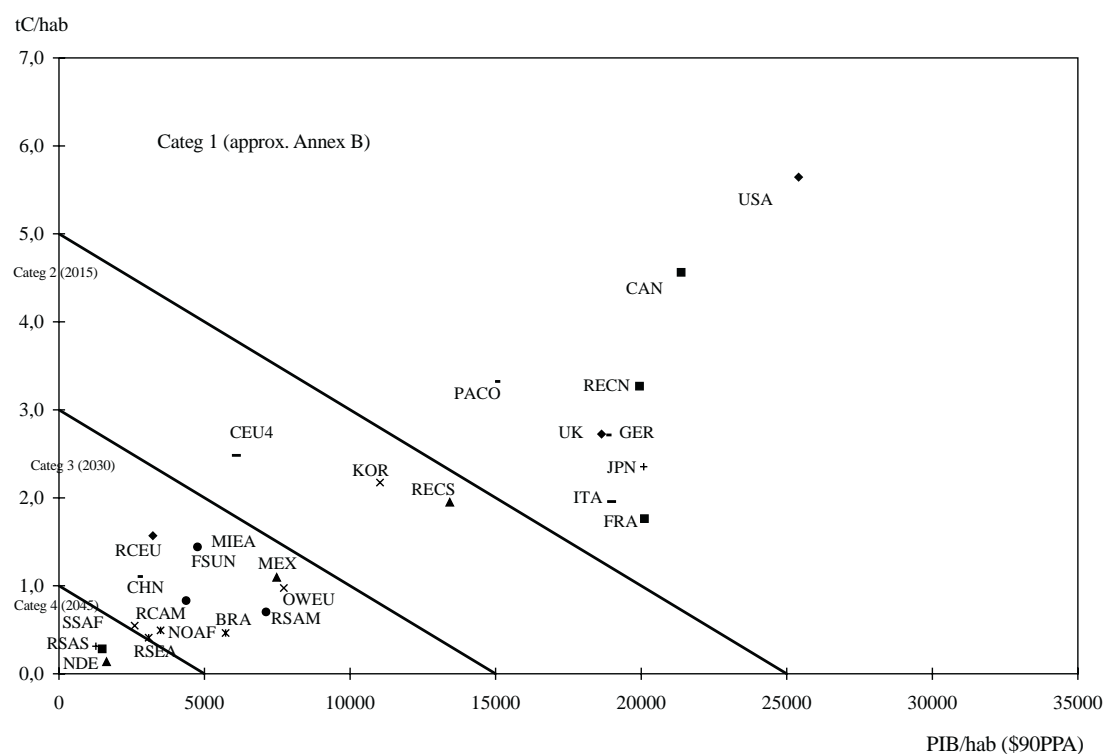


de flexibilité dans le cadre du scénario présenté précédemment.

Pour ce faire, nous construirons d'abord le cadre analytique pour les marchés de droits d'émission : celui-ci est fondé en particulier sur l'analyse de courbes de coûts marginaux de réduction par région, produites par un modèle d'équilibre partiel du secteur énergétique mondial, le modèle POLES. Puis nous fournirons de premiers éléments d'analyse quantitative.

\section{Méthodologie d'analyse des coûts de réduction}

Deux grands types de méthodologies sont utilisés pour évaluer les coûts de réduction des émissions (Grubb et alii,1993 ; chapitre 8 IPCC, 1996) :

- l'approche dite top-down s'appuie sur des modèles macro-économiques, le plus souvent d'équilibre général calculable, avec dans certains cas une représentation détaillée du secteur énergétique ; elle permet notamment de calculer les coûts de réduction en termes de perte de $P I B$ pour différents niveaux de réduction ;

- les études bottom-up, relèvent d'une approche d'ingénieur, ou sont menées avec des modèles d'optimisation du système énergétique ; elles permettent de construire des courbes de potentiel de réduction pour différents niveaux de coût marginal.

L'approche utilisée ici correspond à une démarche intermédiaire puisqu'elle s'appuie sur un modèle mondial d'équilibre sectoriel pour le système énergétique, le modèle POLES (encadré 3).

L'évaluation des coûts de réduction est, dans ce modèle, menée à partir de l'introduction d'une « taxe carbone fictive » ou encore d'une «valeur du carbone » dans l'ensemble des modules de consommation-transformation de l'énergie. Cette taxe carbone fictive entraîne des ajustements de la demande d'énergie finale, par des changements technologiques ou de comportements implicites, ainsi que des substitutions dans les systèmes de conversion d'énergie, pour lesquels les technologies
Encadré 2 : «Stabilisation mondiale à 10 GtC en 2030 »

Emissions mondiales :

$E_{2030} \quad E_{B, 2030} \quad E_{N B, 2030}$ et $E_{2030} \quad 10 G t C$

où $E_{2030}$ représente les émissions mondiales en 2030, $E_{B, 2030}$ les émissions des pays annexe B en 2030 et $E_{N B, 2030}$ les émissions des pays non annexe B en 2030.

\section{Emissions des pays annexe $B$ :}

$\begin{array}{lll}E_{B, 2030} & e_{i, 2030} \\ e_{i, 2030} & e_{i, 1990} *\left(\begin{array}{ll}1 & r_{i}\end{array}\right) *\left(\begin{array}{ll}1 & r_{i}\end{array}\right)\end{array}$

et, de façon plus générale,

$T$ tel que

$$
T 2030, e_{i, T} \quad e_{i, 1990} * 1 \quad \frac{T 2010}{20} r_{i} *\left(1 \quad r_{i}\right)
$$

où $e_{i, T}$ représente les émissions du pays $i$ à l'année $T, r_{i}$ le taux de réduction des émissions tel qu'accepté par le pays $i$ dans le Protocole de Kyoto (s'il s'agit d'un taux d'augmentation des émissions, $r_{i}$ est alors négatif; ceci est le cas par exemple pour l'Australie)

Emissions des pays non annexe $B$ :

$E_{N B, 2030} \quad e_{j, 2030}$

$T$ tel que $2011 T T^{*}$,

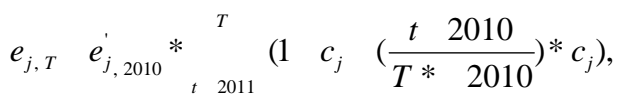

et $\quad T \quad T^{*}, e_{j, T} \quad e_{j, 2010}^{*}{ }_{t 2011}^{T^{*}}\left(1 \quad c_{j} \quad\left(\frac{t \quad 2010}{T * 2010}\right) * c_{j}\right)$

où $e_{j, T}$ représente les émissions du pays $j$ l'année $T, T^{*}$ l'année de stabilisation, $c_{j}$ le taux de croissance des émissions du pays $\mathrm{j}$ en 2010 .

$c_{j}$ est tel que $1 \quad c_{j} \quad(1 \quad 3 \%)\left(1 \quad d_{j}\right)$, où $d_{j}$ représente le taux de croissance démographique moyen annuel du pays $j$ sur la période 2000-2010.

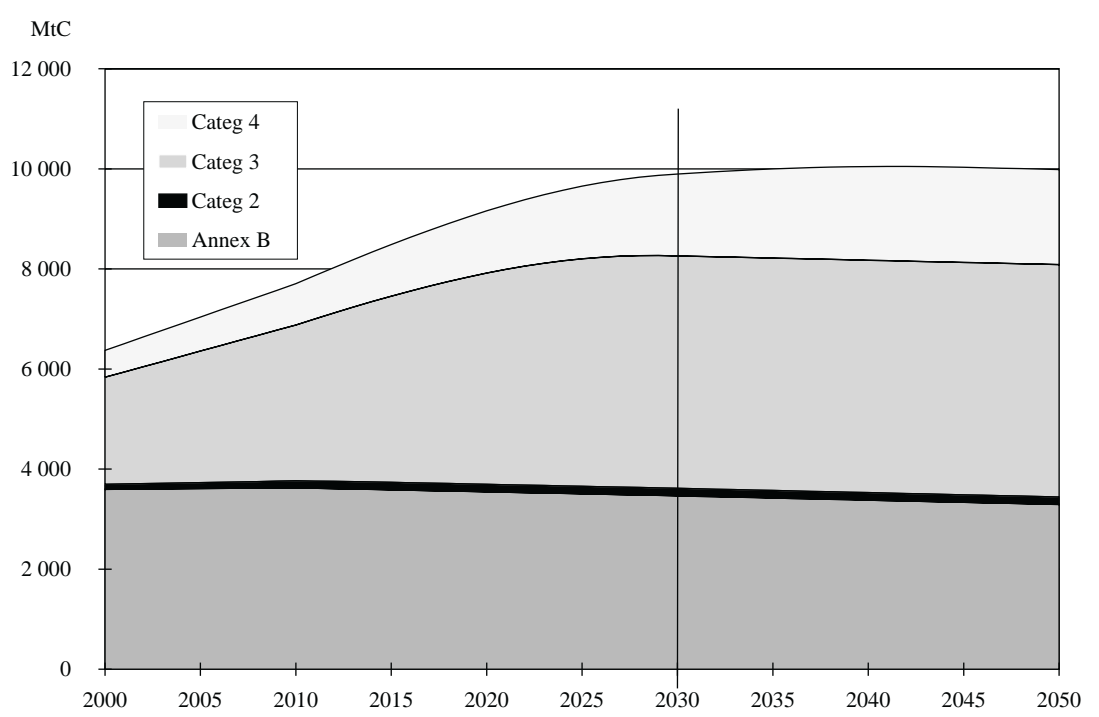




\section{Encadré 3 : le modèle POLES}

Le modèle POLES est un modèle de simulation du système énergétique mondial à l'horizon 2030 : les évolutions démographiques et économiques, pour chacun des grands pays ou régions du monde, sont considérées comme exogènes ; en revanche, les évolutions de l'ensemble des variables caractérisant la consommation, la transformation, la production et les prix de l'énergie sont endogènes au modèle (Criqui et alii, 1996).

Ses principales utilisations relèvent de trois grands types d'exercices :

- Prospective détaillée du système énergétique mondial, par grand pays ou région, avec simulation de la demande par secteur, des technologies liées aux énergies nouvelles et renouvelables, des systèmes électriques, de l'offre et des prix des hydrocarbures.

- Analyse du progrès technique du secteur de l'énergie, soit avec simulation de scénarios exogènes de "percée technologique ", soit par une endogénéisation des effets cumulatifs d'expérience et d'apprentissage sur les nouvelles technologies.

- Simulation des impacts potentiels de la prise en compte des contraintes d'environnement global, exprimées dans les accords internationaux sur la limitation des gaz à effet de serre, avec calcul des Coûts Marginaux de Réduction par pays et par secteur et utilisation du logiciel spécialisé ASPEN (Analyse des Systèmes de Permis d'Emission Négociables) pour la simulation de "marchés de droits d'émission ", éventuellement régulés (Criqui et alii, 1999; Blanchard et alii, 2000 ; Criqui et alii, à paraître).

C'est un modèle de simulation récursive : la dynamique est donnée, à partir du point initial, puis d'année en année, par les ajustements progressifs des variables d'offre et de demande d'une part et de prix d'autre part.
L'horizon prospectif a été volontairement limité à une trentaine d'années, dans le souci de conserver dans le modèle des technologies «explicites» et non «génériques » : hypothèse est faite que les technologies qui pourront avoir un impact quantitatif significatif en 2030 doivent être au moins identifiées aujourd'hui.

Le modèle est construit selon une structure hiérarchisée de modules, interconnectés au niveau national, régional et mondial :

- le plan national intègre les modules de production d'énergies fossiles, de conversion en électricité, d'énergies nouvelles et de consommation par secteurs (10 secteurs pour les pays à modèle détaillé) ;

- les plans régionaux ou mondiaux intègrent les flux d'échanges énergétiques et les modules de prix internationaux, pour les trois grandes énergies faisant l'objet d'un large commerce international, pétrole, gaz naturel et charbon.

Dans sa désagrégation géographique actuelle, le modèle distingue 30 pays ou régions. Par ailleurs, au sein de chaque région, les plus grands pays - ceux du Groupe des 7 (G7) et les cinq grandes économies en développement rapide Chine, Inde, Corée du Sud, Brésil et Mexique - sont identifiés et traités par des modèles détaillés. Cette caractéristique est particulièrement importante pour l'étude des coûts des stratégies de réduction des émissions de gaz à effet de serre, ainsi que des enjeux associés dans la négociation internationale sur le changement climatique.

Une autre caractéristique du modèle POLES est d'offrir une désagrégation détaillée des secteurs et technologies énergétiques, ce qui permet de rattacher toutes les évaluations des coûts marginaux de réduction à des variables technologiques explicites. sont au contraire explicitées. À partir d'une projection de référence dans laquelle la taxe fictive est considérée comme étant égale à zéro, il est ensuite possible de calculer, par simulations successives, les niveaux d'émission associés à une taxe fictive variant pas par pas, de 0 à $600 \$ / \mathrm{tC}$ par exemple. On en déduit ainsi le coût marginal de réduction associé à un niveau donné d'émissions (Criqui et alii, 1997).

L'approche retenue n'est pas exempte de limites. En premier lieu, elle rencontre les difficultés communes à toutes les modélisations prospectives, dans la définition des spécifications et des hypothèses permettant de construire la projection de référence. En second lieu, les coûts ainsi calculés sont des coûts sectoriels ou encore des coûts bruts et ils ne prennent pas en compte l'ensemble des impacts des politiques de réduction.

Principalement, celles-ci se traduiront d'abord par un renchérissement des prix ou des coûts de l'énergie. L'impact macro-économique de ce renchérissement ne serait pas cependant comparable à celui d'un choc pétrolier, puisque les revenus correspondants s'ils existent c'est-à-dire s'il y a taxe ou vente aux enchères de permis d'émission resteraient au sein de chaque économie nationale, où ils pourraient être « recyclés ».

Les «modèles d'équilibre général calculable» permettent de traiter les questions associées, en particulier celle du recyclage et des conditions d'existence d'un «double dividende », écologique et économique. Mais les résultats de ces modèles peuvent manquer de transparence et présenter une moindre robustesse, puisque le calcul du coût net des politiques de réduction combine l'incertitude sur l'évaluation des coûts sectoriels et celle sur les effets macro-économiques directs et de recyclage.

La faiblesse de l'approche sectorielle peut alors aussi constituer sa force. L'identification des coûts sectoriels dans un modèle énergétique permet en effet de traiter la première question de manière approfondie et à l'aide d'un outil adapté, en laissant ouverte la possibilité d'une étude ultérieure de l'utilisation des recettes fiscales ou des revenus de la vente de permis. 
Les marchés de permis d'émission négociables : quelles conséquences pour l'efficacité ?

Le tableau 3 présente les principales implications économiques du scénario «stabilisation à 2030 », si aucun dispositif de flexibilité n'était introduit.

La réduction des émissions mondiales s'élèverait donc à $3600 \mathrm{MtC}$ par rapport à la situation de référence, les pays de l'actuelle Annexe B participeraient pour $1800 \mathrm{MtC}$ soit $50 \%$ du total des réductions d'émissions, pour un coût total qui représenterait $60 \%$ du coût total de la réduction. On peut noter, à partir du tableau 3, trois points importants :

- dans les pays de l'annexe B, le coût brut sectoriel annuel du respect des engagements à 2030 représenterait de 0,005 à $1,7 \%$ du $P I B$; pour les pays non-annexe $\mathrm{B}$, de 0 à $1,6 \%$;

- le coût marginal des réductions projetées varie considérablement d'une région à l'autre, jusqu'à dépasser dans certains cas extrêmes $600 \$ / \mathrm{tC}$, les valeurs hautes étant atteintes en particulier au Europe et au Japon, mais aussi dans certaines régions en développement ;

- le « taux d'effort » (coût de réduction / PIB) serait plus élevé aux États-Unis qu'en Europe, ce qui illustre une fois de plus la nécessité de prendre en compte le coût marginal et les quantités en cause dans l'appréciation des coûts des politiques de réduction ; dans la projection proposée, les États-Unis représenteraient $24 \%$ des réductions en volume et supporteraient $24 \%$ des coûts totaux.

Tableau 3 : le scénario « Stabilisation 2030 » sans flexibilité, une tentative de chiffrage à partir du modèle POLES

\begin{tabular}{|c|c|c|c|c|c|c|}
\hline STABIL 2030 & $\begin{array}{c}\text { Emissions } 2030 \\
\text { Référence } \\
\text { (MtC) }\end{array}$ & $\begin{array}{c}\text { Emissions } 2030 \\
\text { Scénario stabilisation } \\
\text { (MtC) }\end{array}$ & $\begin{array}{l}\text { Réduction } \\
\text { d'émissions } \\
\text { (MtC) }\end{array}$ & $\begin{array}{l}\text { Coût marginal } \\
\text { de réduction } \\
(\$ / \mathbf{t C})\end{array}$ & $\begin{array}{l}\text { Coût total de } \\
\text { réduction } \\
(\mathrm{M} \$)\end{array}$ & \%du PIB \\
\hline USA & 2010 & 1153 & 857 & 246,0 & 76398 & 0,596 \\
\hline Canada & 179 & 102 & 76 & 325,7 & 6937 & 0,586 \\
\hline Mexique & 267 & 235 & 32 & 159,2 & 2475 & 0,101 \\
\hline Reste Am. Cent. & 130 & 84 & 46 & $(>600,0)$ & 8163 & 1,628 \\
\hline Brésil & 339 & 210 & 129 & 338,9 & 15709 & 0,491 \\
\hline Reste Am.Sud & 217 & 213 & 4 & 10,5 & 20 & 0,001 \\
\hline France & 154 & 96 & 58 & 465,2 & 9576 & 0,453 \\
\hline Allemagne & 303 & 193 & 109 & 135,3 & 6086 & 0,227 \\
\hline Italie & 127 & 94 & 33 & 590,0 & 6981 & 0,384 \\
\hline Royaume-Uni & 187 & 130 & 57 & 222,8 & 4639 & 0,243 \\
\hline Reste UE nord & 234 & 134 & 100 & $(>600,0)$ & 18566 & 1,111 \\
\hline Reste UE sud & 172 & 97 & 75 & 430,0 & 8124 & 0,530 \\
\hline Reste Eur. Ouest & 44 & 20 & 24 & $(>600,0)$ & 3484 & 1,278 \\
\hline Turquie & 146 & 120 & 26 & 49,5 & 552 & 0,029 \\
\hline Egypte & 76 & 52 & 24 & 314,2 & 2943 & 0,257 \\
\hline MarocTunisie & 37 & 26 & 11 & 490,0 & 2147 & 0,325 \\
\hline Alg. Libye & 59 & 48 & 11 & 213,2 & 957 & 0,182 \\
\hline Golfe & 366 & 386 & -20 & 0,0 & 0 & 0,000 \\
\hline Moy. Or. Méd. & 95 & 74 & 21 & 201,2 & 1942 & 0,234 \\
\hline Af. Subsaharienne & 784 & 651 & 134 & 51,2 & 3788 & 0,138 \\
\hline Eur. Cent. 4 & 338 & 156 & 182 & 311,9 & 20597 & 1,712 \\
\hline Reste Eur. Cent. & 129 & 94 & 35 & 464,8 & 12570 & 1,766 \\
\hline Eur. cent. NB & 55 & 41 & 14 & 95,8 & 535 & 0,251 \\
\hline Ex Urss B & 834 & 799 & 35 & 10,3 & 179 & 0,005 \\
\hline Ex Urss NB & 209 & 163 & 45 & 152,4 & 2647 & 0,286 \\
\hline Inde & 1044 & 728 & 316 & 148,8 & 22161 & 0,362 \\
\hline Rest e Asie Sud & 209 & 151 & 58 & 298,4 & 6941 & 0,307 \\
\hline Corée Sud & 206 & 149 & 57 & 268,7 & 5821 & 0,486 \\
\hline Reste Asie SE & 897 & 562 & 335 & 295,7 & 33058 & 0,411 \\
\hline Chine & 2788 & 2226 & 562 & 53,1 & 13887 & 0,074 \\
\hline Japon & 365 & 262 & 103 & 340,0 & 13208 & 0,322 \\
\hline Reste Pacifique & 170 & 89 & 81 & 255,9 & 6204 & 0,598 \\
\hline Monde & 13170 & 9540 & 3630 & & 317297 & 0,347 \\
\hline
\end{tabular}

Les coûts sont exprimés en dollars constants de 1990 (1 \$1999=0,82 \$1990).

Source : Modèle POLES et logiciel ASPEN, mars 2000. 
L'étude de ce taux d'effort pour différents pays fait apparaître des situations apparemment paradoxales : il est parfois élevé, soit dans des pays qui sont les plus « vertueux » en termes d'émissions, soit inversement dans les pays moins vertueux que les autres. Ce paradoxe s'explique en fait par les phénomènes suivants :

- dans les pays vertueux, les réductions seront limitées en quantité mais elles se feront à un coût marginal élevé; c'est ce que l'on pourrait qualifier de « syndrome norvégien»;

- dans les pays moins vertueux au contraire, les réductions pourront être obtenues à un coût faible mais elles seront plus importantes en volume; il s'agirait ici du « syndrome australien ».

Il convient donc bien de tenir compte de l'effet quantité et de l'effet coût marginal sur le montant total des programmes de réduction. On peut d'ailleurs noter que les résultats des négociations de Kyoto reflétaient cette réalité, puisque de fait des pays aussi différents que la Norvège et l'Australie sont parvenus à obtenir des objectifs moins contraignants que ceux des autres pays de l'OCDE.

\section{Un marché de droits généralisé aux pays en développement}

À partir de cette situation sans aucune hypothèse de flexibilité, il est maintenant possible d'analyser les gains potentiels de l'introduction d'un système d'échange de permis d'émission négociables, dont les gains d'efficacité économique découlent de la diversité-même des Coûts Marginaux de Réduction constatés dans les différentes régions (voir encadré 4).

Par rapport à la configuration précédente «Stabilisation à $10 \mathrm{GtC}$ au niveau mondial en 2030, sans échange », l'introduction d'un marché de droits produit une situation sensiblement différente (tableau 4).

L'objectif quantitatif est toujours celui d'une réduction totale d'environ $3600 \mathrm{MtC}$ par rapport à la référence de 2030 pour le monde, alors même que les droits initiaux (2010) des pays en développement correspondent à un doublement de leurs émissions par rapport à 1990. Le coût marginal mondial du respect des objectifs ou encore le prix des permis est alors de $127,5 \$ / \mathrm{tC}$.

Dans ce schéma d'allocation mondiale, seuls 7 pays (ou groupes de pays) seraient vendeurs de permis, les principaux étant la Chine, la Russie, l'Afrique subsaharienne et les pays du Golfe. Les échanges atteignent près de $900 \mathrm{MtC}$, ce qui correspond à $25 \%$ de la réduction des émissions, pour un montant total d'environ 114 Mrds\$. Les principaux acheteurs de permis seraient les États-Unis avec $235 \mathrm{MtC}$ (30 Mrds\$), mais il faut aussi noter la présence d'acheteurs des pays en développement pour près de

\section{Encadré 4 : la simulation des échanges de permis d'émission, dans des marchés ou «bulles »}

Les courbes de coûts marginaux de réduction (CMR) peuvent être étudiées pour des régions isolément, mais aussi pour un ensemble consolidé : le principe de base est alors que, pour une valeur du carbone donnée, les réductions d'émission d'une région $A$ et d'une région $B$ sont additives pour calculer les réductions d'émission - et consécutivement les courbes de coûts marginaux de réduction - de la région consolidée $\mathrm{A}+\mathrm{B}$.

Cette propriété ouvre la voie à l'analyse de bulles ou de marchés, au sein desquels il y aurait une égalisation des coûts marginaux de réduction, et donc minimisation du coût total, par échange de permis d'émission notamment. Le graphique ci-dessous décrit la configuration de base pour l'échange de droits d'émission entre deux pays. La situation représentée est celle de deux pays soumis à des contraintes d'émission, par exemple deux pays industrialisés de l'annexe B : en abscisse figurent les réductions d'émission à effectuer $\left(Q_{A}\right.$ pour le pays $\mathrm{A}, Q_{B}$ pour le pays B et $Q_{A}+Q_{B}$ pour la zone consolidée) et en ordonnée les coûts marginaux ; le coût marginal de réduction pour la zone consolidée ou encore la valeur des permis s'établit à un niveau intermédiaire entre les coûts marginaux des deux pays pris individuellement; le montant total des échanges de permis correspond à la surface grisée sur la figure et les gains de chaque partenaire à la différence entre d'une part le coût total sans permis et d'autre part le coût des réductions internes plus les achats ou moins les ventes de permis.

Principe de l'échange de permis d'émission entre deux pays contraints

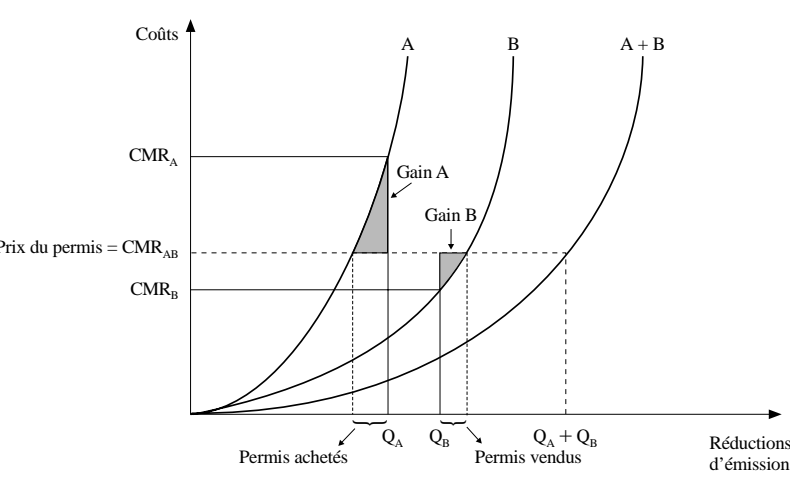

Toutes les études menées à l'aide du modèle POLES concernent une analyse des courbes de coûts marginaux de réduction à l'horizon 2030, en statique. Ces courbes supposent que la valeur du carbone correspondante (ou encore la taxe carbone fictive) est introduite dès l'année 2000. Une nouvelle version du modèle est actuellement en développement. Elle permettra d'analyser, en dynamique, l'évolution des valeurs du carbone, année après année. 
$300 \mathrm{MtC}$ (Amérique Centrale, Brésil, Afrique du Nord, Asie hors Chine).

Le principal résultat de cet exercice est sans doute que le coût total du programme de stabilisation des émissions est ramené de plus de 300 Mrds de $\$$ dans le cas sans flexibilité à environ 200 Mrds \$ par an. Les gains nets par rapport à la situation hors marché sont importants pour certaines régions : $73 \mathrm{Mds}$ de $\$$ pour les pays acheteurs (dont 12 pour les États-Unis) et de 44 Mrds de $\$$ pour les pays vendeurs (dont 21.5 pour la Chine), soit un total de 117 Mds \$. Le marché permettrait donc aux pays vendeurs de permis de bénéficier de recettes qui pourraient financer, et au delà, les coûts des réductions supplémentaires qu'ils effectuent, à supposer cependant que soient mis en place les signaux et mécanismes incitatifs initiaux.

L'ouverture du marché à l'ensemble des pays du globe est donc économiquement plus efficace que la solution sans marché et tous les pays voient leur taux d'effort diminuer de manière significative. Le problème est cependant que les taux de réduction en interne deviennent alors hétérogènes, puisqu'ils oscillent entre $24 \%$ et $97 \%$ (pour l'Allemagne). La France présente un taux de $49 \%$, du fait de coûts marginaux rapidement élevés, en raison d'un profil initial peu intensif en carbone.

Il convient enfin de souligner que le cas présenté demeure largement théorique, puisqu'il suppose,

Tableau 4 : marché de droits d'émission mondial : un prix d'équilibre à 127,5\$/tC

\begin{tabular}{|c|c|c|c|c|c|c|c|c|c|c|c|}
\hline & $\begin{array}{l}\text { Référ. } \\
2030 \\
(\mathrm{MtC})\end{array}$ & $\begin{array}{c}\text { Stabil. } \\
2030 \\
\\
(\mathrm{MtC}) \\
\end{array}$ & $\begin{array}{c}\text { Avec } \\
\text { marché } \\
\text { (MtC) }\end{array}$ & $\begin{array}{c}\text { Réduct. en } \\
\text { interne } \\
\% \\
\end{array}$ & \begin{tabular}{|c} 
Volume des \\
échanges \\
(MtC) \\
\end{tabular} & $\begin{array}{c}\text { Valeur des } \\
\text { échanges } \\
\text { (M\$) }\end{array}$ & $\begin{array}{c}\text { Coût } \\
\text { interne } \\
\text { (M\$) }\end{array}$ & $\begin{array}{l}\text { Coût } \\
\text { total } \\
\text { (M\$) }\end{array}$ & $\begin{array}{c}\text { Coût sans } \\
\text { échange } \\
\text { (M\$) } \\
\end{array}$ & $\begin{array}{c}\text { Gains } \\
\text { échange } \\
\text { (M\$) }\end{array}$ & $\begin{array}{c}\text { Coût } \\
\text { après } \\
\text { échange } \\
\text { \% PIB }\end{array}$ \\
\hline USA & 2010 & 1153 & 1388 & 72,6 & $-235,2$ & 29990 & 34393 & 64383 & 76398 & 12014 & 0,502 \\
\hline Canada & 179 & 102 & 123 & 73,0 & $-20,7$ & 2637 & 2538 & 5175 & 6937 & 1762 & 0,437 \\
\hline Mexique & 267 & 235 & 241 & 81,6 & $-6,0$ & 761 & 1620 & 2381 & 2475 & 95 & 0,097 \\
\hline RestAmCen & 130 & 84 & 115 & 33,1 & $-30,5$ & 3884 & 864 & 4748 & 8163 & 3415 & 0,674 \\
\hline Brésil & 339 & 210 & 263 & 58,7 & $-53,4$ & 6811 & 4069 & 10880 & 15709 & 4829 & 0,340 \\
\hline RestAmSud & 217 & 213 & 187 & & 26,2 & -3346 & 1390 & -1956 & 20 & 1976 & $-0,054$ \\
\hline France & 154 & 96 & 125 & 49,4 & $-29,3$ & 3741 & 1476 & 5218 & 9576 & 4358 & 0,247 \\
\hline Allemagne & 303 & 193 & 196 & 97,1 & $-3,2$ & 404 & 5670 & 6074 & 6086 & 12 & 0,227 \\
\hline Italie & 127 & 94 & 114 & 41,3 & $-19,4$ & 2470 & 768 & 3238 & 6981 & 3743 & 0,178 \\
\hline Roy Uni & 187 & 130 & 143 & 76,6 & $-13,4$ & 1706 & 2350 & 4056 & 4639 & 583 & 0,212 \\
\hline RestUEnor & 234 & 134 & 193 & 41,6 & $-58,5$ & 7463 & 2175 & 9637 & 18566 & 8929 & 0,494 \\
\hline RestUEsud & 172 & 97 & 120 & 69,9 & $-22,5$ & 2866 & 2470 & 5335 & 8124 & 2789 & 0,348 \\
\hline RestEuwest & 44 & 20 & 38 & 24,0 & $-18,3$ & 2327 & 350 & 2677 & 3484 & 807 & 0,436 \\
\hline Turquie & 146 & 120 & 103 & & 16,6 & -2111 & 1922 & -189 & 552 & 741 & $-0,010$ \\
\hline Egypte & 76 & 52 & 63 & 56,9 & $-10,2$ & 1294 & 718 & 2012 & 2943 & 931 & 0,175 \\
\hline MarocTuni & 37 & 26 & 33 & 40,3 & $-6,5$ & 832 & 290 & 1121 & 2147 & 1026 & 0,170 \\
\hline AlgLibye & 59 & 48 & 51 & 73,9 & $-2,9$ & 373 & 461 & 834 & 957 & 123 & 0,159 \\
\hline Golfe & 366 & 386 & 315 & & 71,5 & -9112 & 2830 & -6283 & 0 & 6283 & $-0,303$ \\
\hline MoyO Méd & 95 & 74 & 81 & 67,2 & $-6,8$ & 872 & 815 & 1687 & 1942 & 254 & 0,204 \\
\hline Af Subsah & 784 & 651 & 539 & & 112,2 & -14308 & 13171 & -1137 & 3788 & 4925 & $-0,041$ \\
\hline EurCent4 & 338 & 156 & 225 & 61,8 & $-69,5$ & 8860 & 6482 & 15342 & 20597 & 5255 & 1,275 \\
\hline RestEuCent & 129 & 94 & 131 & 58,8 & $-37,0$ & 4714 & 2510 & 7225 & 12570 & 5345 & 1,015 \\
\hline EurcentNB & 55 & 41 & 39 & & 2,0 & -250 & 753 & 503 & 535 & 33 & 0,235 \\
\hline ExUrss B & 834 & 799 & 672 & & 127,1 & -16200 & 7948 & -8253 & 179 & 8432 & $-0,223$ \\
\hline ExUrssNB & 209 & 163 & 168 & 89,6 & $-4,7$ & 602 & 1987 & 2589 & 2647 & 58 & 0,280 \\
\hline Inde & 1044 & 728 & 761 & 89,4 & $-33,7$ & 4291 & 17513 & 21804 & 22161 & 357 & 0,356 \\
\hline RestAsiSud & 209 & 151 & 175 & 57,9 & $-24,3$ & 3104 & 1826 & 4930 & 6941 & 2011 & 0,218 \\
\hline Corée Sud & 206 & 149 & 169 & 65,1 & $-19,8$ & 2530 & 2022 & 4551 & 5821 & 1270 & 0,380 \\
\hline RestAsie SE & 897 & 562 & 670 & 67,5 & $-108,9$ & 13885 & 11047 & 24932 & 33058 & 8127 & 0,310 \\
\hline Chine & 2788 & 2226 & 1684 & & 542,3 & -69143 & 61583 & -7560 & 13887 & 21447 & $-0,041$ \\
\hline Japon & 365 & 262 & 307 & 56,4 & $-45,0$ & 5741 & 3238 & 8980 & 13208 & 4229 & 0,219 \\
\hline RestPacif & 170 & 89 & 107 & 77,5 & $-18,1$ & 2314 & 2860 & 5174 & 6204 & 1030 & 0,499 \\
\hline Monde & 13170 & 9540 & 9540 & - & $(897,9)$ & (114471) & 200108 & 200108 & 317297 & 117189 & 0,212 \\
\hline
\end{tabular}

Les valeurs monétaires sont exprimées en \$ constants de 1990.

Source : Modèle POLES et logiciel ASPEN, mars 2000. 
d'une part, que tous les potentiels de réduction dans les pays en développement peuvent être réalisés et, d'autre part, que les mécanismes de flexibilité fonctionnent parfaitement, c'est-à-dire que l'on se place dans une situation de marché en concurrence pure et parfaite, sans coûts de transaction. Dans cette perspective, la valeur des permis doit être interprétée comme une valeur minimum ou valeur plancher et, inversement, le volume des échanges comme un volume maximum.

\section{Evaluation ex post du scénario en termes d'équité et conditions de son acceptabilité}

Dans la première partie, nous avons souligné la grande difficulté de la construction d'un accord ex ante sur un critère unique d'équité pour l'attribution des droits mondiaux d'émission à l'horizon 2030. C'est la raison pour laquelle nous avons proposé une démarche différente qui consiste dans un premier temps à définir un scénario d'attribution des droits pragmatique, puis à l'évaluer en termes d'équité, à l'aune d'une pluralité de critères renvoyant eux-mêmes à divers principes d'équité.

Il est clair que le scénario de stabilisation mondiale ne sera acceptable par la plupart des Parties que s'il conduit à une certaine convergence pour les critères sur lesquels s'appuient les Parties dans leurs débats sur l'équité. Il convient par conséquent d'analyser ce scénario au moins du point de vue du critère des émissions par habitant (principe d'égalité des droits, défendu par de nombreux pays en développement) et du critère du taux d'effort exprimé en coût des réductions par unité de $P I B$ (principe d'égalité démocratique, défendu par certains pays en développement et fréquemment examiné par les pays de l'annexe B).

Figure 3 : courbes de Lorenz et indices de Gini pour les droits d'émissions mondiaux

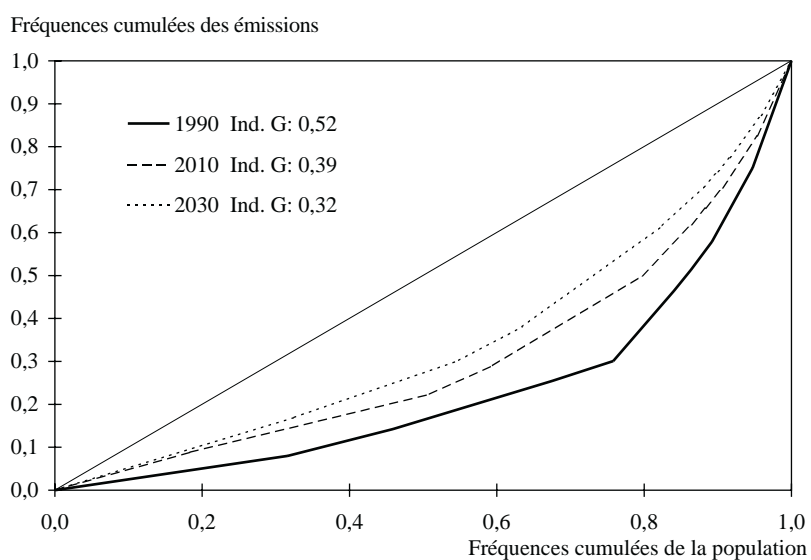

Source : modèle POLES.
Utilisation de l'indice de Gini pour une mesure synthétique de la convergence des émissions par habitant

C'est pour mesurer l'équité dans la répartition des revenus d'une population que la courbe de Lorenz et l'indice de Gini ont été développés initialement. Cependant, il est tout à fait possible de transposer ces outils pour mesurer l'équité dans la répartition des émissions ou des droits d'émission d'une population. La figure 3 présente les courbes de Lorenz pour les années 1990, 2010 et $2030: 1$ 'axe des abscisses correspond aux fréquences cumulées de la population et l'axe des ordonnées aux fréquences cumulées des émissions. La courbe de 1990 représente les émissions réelles de $\mathrm{CO}_{2}$ ou les droits « de fait » de cette année-là, tandis que celle de 2010 décrit la répartition des droits d'émission telle qu'acceptée dans le Protocole de Kyoto. Celle de 2030 résulte de l'attribution des droits décrite dans le scénario «Stabilisation mondiale à $10 \mathrm{GtC}$ en $2030 »$.

Rappelons que lorsque la courbe de Lorenz se confond avec la bissectrice, la répartition est parfaitement égalitaire. La figure 3 fait ainsi apparaître que la situation de fait en 1990 est la plus inégalitaire, que la situation de droit en 2010 l'est moins et que la situation hypothétique de 2030 permet encore de réduire les inégalités dans la répartition des droits d'émission par rapport à celle de 2010. Ces constats sont évidemment confirmés par le calcul de l'indice de Gini ${ }^{(18)}$ : il passe de 0,52 en 1990 à 0,39 en 2010 et 0,32 en 2030 .

En termes d'émissions par habitant, le tableau 5 met en évidence la réduction à laquelle se sont engagés les pays de l'annexe B dans le Protocole de Kyoto (situation 2010) et à laquelle ils seraient soumis dans le scénario de stabilisation. Les émissions des pays non-annexe B peuvent au contraire augmenter, sans contrainte jusqu'en 2010 (Protocole de Kyoto), et de façon plus « encadrée » dans le scénario de stabilisation. Les trajectoires d'émissions par habitant tendent ainsi à se resserrer entre les pays annexe $B$ et non-annexe $B$, vers une convergence plus marquée.

\section{Vers l'égalité démocratique}

Le taux d'effort? consenti par les pays pour réduire leurs émissions (coût total des réductions / PIB), constitue l'un des critères pour évaluer l'équité du scénario de stabilisation en termes d'égalité démocratique. Il est présenté dans les tableaux 3 et 4 , successivement dans une configuration de réduction des émissions sans échange puis dans l'hypothèse d'un marché de permis d'émission fonctionnant parfaitement.

Sans échange, le taux d'effort qui serait engagé par les pays annexe B s'avère généralement plus élevé que celui des pays non-annexe $B$. La règle de 
Tableau 5 : émissions par habitant 1990, 2010, 2030

\begin{tabular}{|l|c|c|c|}
\hline Emissions par habitant (tC) & 1990 & 2010 & 2030 \\
\hline Amérique du Nord & 5,23 & 4,15 & 3,53 \\
Europe de l'Ouest & 2,19 & 1,95 & 1,83 \\
Pacifique OCDE & 2,53 & 2,24 & 2,16 \\
Ex URSS & 3,46 & 2,81 & 2,64 \\
Europe de l'Est & 2,37 & 2,23 & 2,27 \\
Amérique latine & 0,62 & 0,86 & 1,05 \\
Afrique Nord- Moyen-Orient & 0,48 & 0,53 & 0,68 \\
Chine & 0,58 & 1,13 & 1,42 \\
Reste Asie & 0,28 & 0,46 & 0,60 \\
\hline Moyenne mondiale & 1,11 & 1,10 & 1,14 \\
\hline
\end{tabular}

différenciation qui propose un coût de réduction proportionnel au PIB et qui correspondrait au principe d'égalité démocratique est donc globalement atteinte et même dépassée, si l'on s'intéresse aux deux groupes de pays dans leur ensemble (annexe B versus non-annexe B). De façon similaire, celle qui préconise un taux d'effort proportionnel au PIB par habitant et qui correspond au principe de différence de Rawls se trouve respectée dans les grandes lignes.

Cependant, si l'on examine dans le détail, pays par pays, le principe de différence de Rawls n'est pas encore atteint. Au sein des pays de l'annexe B, les pays d'Europe du Nord et d'Europe Centrale par exemple ont un taux d'effort respectivement de $1,1 \%$ et de $1,7 \%$ du PIB, contre $0,6 \%$ pour les États-Unis ou $0,3 \%$ pour le Japon (tableau 3). De même, parmi les pays non- annexe B, les pays d'Amérique Centrale et l'Inde affichent respectivement un taux de $1,6 \%$ et de $0,4 \%$ du $P I B$ contre $0,1 \%$ pour le Mexique et $0,07 \%$ pour la Chine. En conséquence, notre scénario de stabilisation doit être considéré comme un scénario préliminaire qu'il conviendra d'améliorer au cours de recherches ultérieures pour atteindre une convergence plus satisfaisante du point de vue des principes rawlsiens.

Par ailleurs, comme cela a déjà été noté précédemment, l'ouverture du marché des permis d'émission conduit pour chaque pays à une réduction de son taux d'effort, par rapport à une situation sans échange. Cela n'est pas surprenant, dans la mesure où, par l'ouverture du marché, il y égalisation des coûts marginaux de réduction et, par voie de conséquence, minimisation du coût total, avec partage des gains entre acheteurs et vendeurs. Si dans cette configuration on regarde les pays de l' annexe B, les coûts par pays, en proportion du PIB, tendent à une plus grande convergence, ce qui implique une meilleure égalité démocratique associée à une meilleure égalité utilitariste, la réduction relative de l'égalité des émissions effectives par habitant - qui renvoie à l'égalité des droits - étant monétairement compensée par les flux financiers. La question est alors de savoir quelle serait l'acceptabilité de ce scénario, compte tenu de ses impacts différenciés selon les trois critères d'équité examinés ici.

L'approche que nous avons développée a donc permis une première quantification des enjeux associés à la mise en œuvre de marchés internationaux de droits d'émission pour l'horizon post-2010 et au plan mondial. Il ne s'agit ici que d'une première étape dans la recherche dont les résultats restent provisoires et devront être affinés. Ces résultats permettent cependant de tirer un certain nombre de conclusions analytiques sur les voies possibles de l'insertion des pays en développement dans un système mondial d'attribution des droits ainsi que sur la structure et l'ordre de grandeur des flux associés à la mise en œuvre des marchés.

Le principe de l'introduction de mécanismes de flexibilité est-il susceptible d'être remis en cause par l'analyse, a posteriori, de la répartition des avantages qui en découlent? Nous ne le pensons pas, car si cette répartition apparaissait par trop déséquilibrée, il faudrait alors plutôt reconsidérer le scénario d'attribution des droits en amont. Ainsi, l'enjeu des négociations à venir sera la construction - dans une procédure itérative - d'un accord sur les règles génériques d'attribution, auxquelles devront être associés des dispositifs de différenciation.

Dans cette perspective, notre premier exercice constitue une voie possible pour les négociations, mais il fait apparaître des situations déséquilibrées en défaveur de certaines régions comme l'Inde ou l'Amérique Centrale, ce qui nécessitera sans doute un affinement des hypothèses de détail dans les scénarios à venir. Il faudra notamment modifier la catégorisation des pays proposée dans notre annexe, pour prendre en compte la spécificité de certains pays en développement (et donc leurs délais de stabilisation).

Ce premier exercice ouvre ainsi sur la construction et l'analyse d'autres scénarios selon, en particulier, les critères d'égalité des droits et d'égalité démocratique, sachant que l'égalité utilitariste est en théorie garantie par l'introduction du marché des droits. Plus les scénarios permettront d'assurer un équilibre satisfaisant de ces différents points de vue sur l'équité, plus ils seront acceptables et plus grandes seront les chances de parvenir à un accord sur le programme de stabilisation des émissions à 2030 qui s'impose dans une perspective à plus long terme de stabilisation des concentrations. 


\section{Conclusion générale}

Par le Protocole de Kyoto, la répartition des permis d'émission au sein des Parties de l'annexe B est désormais établie pour l'horizon 2008-2012. Le recours aux dispositifs de flexibilité et aux permis d'émission négociables est aussi prévu - quoique dans des conditions qui demeurent encore à préciser - pour les pays soumis à des engagements de réduction contraignants. En revanche, la question d'un engagement futur des pays en développement reste totalement ouverte, avant la Conférence des Parties de La Haye.

La réduction des émissions, obtenue dans le Protocole de Kyoto pour les pays de l'annexe B, ne représente qu'un faible volume face aux augmentations prévues sur la même période dans les pays en développement. La participation de ceux-ci à des engagements contraignants de limitation des émissions semble donc nécessaire à terme, pour atteindre l'objectif de stabilisation de la concentration des gaz à effet de serre dans l'atmosphère. Par conséquent, les questions de la fixation des objectifs quantitatifs et de la différenciation vont resurgir inéluctablement, pour l'insertion des pays en développement, mais aussi pour les objectifs post-2010 des pays constituant aujourd'hui l'annexe B.

Dans ce contexte, notre papier a développé certains éléments méthodologiques pouvant contribuer à une double réflexion, sur les voies équitables d'une insertion des pays en développement dans un programme mondial à long terme de limitation de la croissance des émissions, et sur les apports, en termes d'efficacité économique des systèmes de permis d'émission négociables.

Sur le premier point, il s'avère qu'aucune règle opératoire de différenciation, ni aucun principe unique d'équité ne semblent pouvoir obtenir un consensus de tous les pays, dans la mesure où les intérêts demeurent structurellement divergents. De ce fait, nous avons construit un scénario simple et pragmatique de stabilisation des émissions de $\mathrm{CO}_{2}$ à l'horizon 2030 et montré ensuite qu'il pourrait conduire à une relative convergence des droits et à un meilleur respect de l'égalité démocratique.

Sur le plan de l'efficacité, les résultats obtenus soulignent l'intérêt que revêt l'instauration d'un marché de permis d'émission négociables, puisqu'il permettrait de rétablir expost l'efficacité qui n'existe pas a priori dans la répartition initiale des droits. La conception utilitariste de l'équité serait alors aussi respectée.

À partir des conclusions de cette étude, on peut affirmer que les solutions qui seront élaborées dans le cours futur du processus de négociation ne pourront durablement ignorer trois nécessités : celle d'un certain degré de convergence progressive sur le long terme des droits d'émission par habitant-pour l'équité internationale des droits -, celle de l'acceptabilité des coûts imposés aux différentes régions du monde - pour l'équité dans l'effort-, enfin celle de l'instauration de mécanismes larges de flexibilité - pour l'efficacité globale de cet effort. 


\section{Notes}

(1) Quantitative Emission Limitation and Reduction Objectives.

(2) Au 13 janvier 2000, 84 Parties de la Convention ont signé le Protocole et 22 l'ont ratifié.

(3) La cinquième Conférence des Parties (COP 5, Bonn, novembre 1999) a essentiellement poursuivi les travaux de COP 4 en mettant l'accent sur la nécessité d'intensifier les négociations d'ici COP 6.

(4) Selon les projections du modèle POLES, il faut compter avec un doublement ou un triplement des émissions des pays en développement d'ici 2010.

(5) Tout d'abord, il s'agit d'objectifs d'évolution des émissions, dans le sens où il n'est pas question de réduction des émissions mais de moindre croissance des émissions, pour les pays en développement. De plus, les «émissions » considérées dans ce texte concernent le seul $\mathrm{CO}_{2}$ et non les émissions des 5 autres gaz à effet de serre retenus également dans le Protocole de Kyoto.

(6) Il s'agit par exemple du Japon, ou du Brésil.

(7) Pour plus de détails sur les principales règles de différenciation proposées (définitions, critères de différenciation, limites, pays ou études les mentionnant), voir Blanchard et alii, (1998).

(8) Bien que le terme de « réduction d'émissions » ne soit pas approprié pour les pays en développement, il sera employé ici pour rester fidèle aux études et aux propositions des pays (élaborées pour la plupart en vue d'une différenciation au sein des seuls pays industrialisés).

(9) L'exemple de l'Iran est significatif à cet égard : ce pays propose d'établir une différenciation basée à la fois sur le $P I B$, la part historique, la dépendance vis-à-vis des combustibles fossiles, l'accès aux énergies renouvelables, le budget de la défense, la croissance démographique, les circonstances spéciales et la part dans le commerce international (Reiner et alii, 1997).

(10) Le Global Commons Institute qualifie cette règle de : « contraction et convergence »(GCI, 1996). Agarwal et Narain sont parmi les premiers, avec le GCI, à l'avoir proposée Agarwal et alii (1991). Ils développent leur approche dans Agarwal (1998) et Agarwal et alii (1998).

(11) Le Pew Center on Global Climate Change (Claussen et alii, 1998) évoque la possibilité de prendre en compte les émissions futures (et non plus passées) dans la mesure de la responsabilité des pays. Sans compter la polémique sur la façon de mesurer ces émissions, le résultat en termes de différenciation serait opposé et non acceptable par les pays en développement.

(12) Sur cette question, voir la synthèse réalisée par P. Hassner dans le Dictionnaire d'éthique et de philosophie morale (1996, pp. 1278-85) et le commentaire de S. Hoffmann sur le Droit des gens de Rawls (Rawls, 1996).

(13) Sur ce point, voir notamment : Godard (1992) ; Rose (1992); Grubb (1995) et Paterson (1996).

(14) Paterson fait exception puisqu'il établit une relation entre la théorie du droit de Nozick et la règle du statu quo (Paterson, 1996, note de bas de page 68).

(15) Ppmv : particules par million de volume.
(16) Exemples : chapitre 8, IPCC (1996); Gupta et alii (1999)

(17) On notera qu'un taux de croissance des émissions par habitant de $3 \%$ revient à une croissance de $3 \%$ du PIB par habitant, à intensité constante des émissions par unité de $P I \mathrm{~B}$. En effet, comme on peut écrire $\mathrm{E} / \mathrm{POP}=(\mathrm{E} / P I B) *(P I B / \mathrm{POP})$, il $\mathrm{y}$ a en première approximation additivité des taux de croissance de E/PIB et de $P I B / P O P$, pour de petites variations.

(18) Rappelons que l'indice de Gini est le rapport entre la surface de concentration (surface comprise entre la courbe de Lorenz et la bissectrice) et la surface du triangle qui coupe le rectangle des fréquences en 2 par la bissectrice; l'indice est donc compris entre 0 pour une répartition parfaitement égalitaire et 1 pour une répartition parfaitement inégalitaire. 


\section{Bibliographie}

Agarwal A. (1998). « Attribution des quotas : équité ou loi du plus fort ? ", Courrier de la Planète / Global Chance, mars-avril, pp. 31-32.

Agarwal A. et Narain S. (1991). Global Warming in an unequal world, New Delhi : Centre for Science and Environment.

Agarwal A. et Narain S. (1998). The Atmospheric Rights of all People on Earth, New Delhi : Centre for Science and Environment.

Audard C. (1996). «Utilitarisme », in : M. Canto-Sperber (ed.), Dictionnaire d'éthique et de philosophie morale, Paris : Presses Universitaires de France.

Bentham J. (1789). An Introduction to the Principles of Morals and Legislation, Oxford : Blackwell, 1948.

Blanchard O., Criqui P., Trommetter M., Viguier L. (1998). Différenciation, équité internationale et efficacité dans la lutte contre le changement climatique global, Communication aux Journées AFSE 1998 sur l'Economie de l'environnement et des ressources naturelles, Toulouse, 11-12 mai 1998, 33 p. (Grenoble : IEPE, Cahier de recherche de l'IEPE $\left.n^{\circ} 14\right)$.

Blanchard O., Criqui P. (2000). « La valeur du carbone : un concept générique pour les politiques internationales et nationales de réduction des émissions ", Economie Internationale, $\mathrm{n}^{\circ} 82$, pp. $75-102$

Claussen E., McNeilly L. (1998). The Complex Elements of Global Fairness, Pew Center on Global Climate Change, Washington DC, October 29, $36 \mathrm{p}$.

Criqui P. et al. (1996). POLES 2.2., JOULE II Programme, European Commission DG XVII - Science Research Development, Bruxelles, December, 99 p.

Criqui P., Kouvaritakis N. (1997). Les coûts pour le secteur énergétique de la réduction des émissions de $\mathrm{CO}_{2}$ : une évaluation internationale avec le modèle POLES, Grenoble : IEPE, Cahier de Recherche de l'IEPE n 13, 20 p.

Criqui P., Mima S., Viguier L. (1999). " Marginal Abatement Costs of $\mathrm{CO}_{2}$ Emission Reductions, Geographical Flexibility and Concrete Ceilings : an Assessment Using the POLES Model », Energy Policy, October 27, pp. 585-601.

Criqui P., Viguier L. (à paraître). « Régulation des marchés de droits d'émission négociables pour le $\mathrm{CO}_{2}$ : une proposition de plafonds pour les quantités et pour les prix ».

Dupuy J. P. (1992). Libéralisme et justice sociale, Paris : Hachette Littérature.

Energy Journal, special issue (1999). The Costs of the Kyoto Protocol : a Multi-Model Evaluation.

Fishkin J. (1986). « Theories of Justice and International Relations : the Limits of Liberal Theory ", in : A. Ellis, Ethics and international relations, Manchester : Manchester University Press.

Framework Convention on Climate Change (FCCC), Ad Hoc Group on the Berlin Mandate (1996), Strengthening the Commitments in Article 4.2 (A) and (B) : Quantified Emissions Limitation and Reduction Objectives within Specified Time-Frames : Review of Possible Indicators to Define Criteria for Differentiation Among Annex I Parties, Note by the Secretariat, FCCC/AGBM/1996/7, 21 juin.
Gauthier D. (1986). Morals by Agreement, Oxford : Oxford University Press.

Global Commons Institute (GCI) (1996). Draft Proposals for a Climate Change Protocol Based on Contraction and Convergence, a Contribution to Framework Convention on Climate Change, Ad Hoc Group on the Berlin Mandate (AGBM/1.9.96/14), $6^{\text {th }}$ september.

Godard O. (1992). «Des marchés internationaux de droits à polluer pour le problème de l'effet de serre : de la recherche de l'efficacité aux enjeux de légitimité ", Revue Politiques et Management Public, vol. 10, $\mathrm{n}^{\circ}$ 2, juin, pp. 101-131.

Godard O. (1997). « De Rio à Kyoto : pourquoi la convention sur le climat devrait intéresser ceux qui ne s'intéressent pas au climat », Futuribles, n 224, pp. 31-68.

Grubb M., Edmonds J., ten Brink P., Morrison M. (1993). «The Costs of Limiting Fossil-Fuel $\mathrm{CO}_{2}$ Emissions, a Survey and Analysis ", Annual Review of Energy and Environment, vol. 18 , pp. $397-478$.

Grubb M., (1995). « Seeking Fair Weather : Ethics and the International Debate on Climate Change ", International Affairs, vol. 71, n 3, pp. 463-496.

Gupta S., Bhandari P.M. (1999). « An Effective Allocation Criterion for $\mathrm{CO}_{2}$ Emissions », Energy Policy, n ${ }^{\circ} 27$, pp. 727-736.

Hassner P. (1996). "Relations internationales », in : M. Canto-Sperber (ed.), Dictionnaire d'éthique et de philosophie morale, Paris : Presses Universitaires de France.

IPCC (Intergovernmental Panel on Climate Change) (1996). Climate Change 1995 - Economic and social dimensions of climate change, Contribution of Working Group III to the Second Assessment Report of the Intergovernmental Panel on Climate Change, Cambridge University Press, Cambridge.

Locke J. (1690). Traité du gouvernement civil, Paris : Flammarion, 1992.

Marx K. (1875). « Gloses marginales au programme du Parti ouvrier Allemand », in : K. Marx et F. Engels, eds, Critique des programmes de Gotha et d'Erfurt, Paris : Editions Sociales, 1996.

Müller B. (1998). Justice in Global Warming Negociations : how to Obtain a Procedurally Fair Compromise, Oxford Institute for Energy Studies, december, 83 p. (EV26).

Nozick R. (1996). Anarchy, State, Utopia, Oxford : Blackwell.

Paterson M. (1996). «International Justice and Global warming », in : B. Holden, ed., The Ethical Dimensions of Global Change, McMillan.

Pigou A. C. (1932). The Economics of Welfare, London : Macmillan, 4e éd.

Rawls J. (1971). A Theory of Justice, Cambridge : Harvard University Press.

Rawls J. (1996). Le droit des gens, Paris : Editions Esprit.

Reiner D.M., Jacoby H.D. (1997). Annex I Differentiation Proposals : Implications for Welfare, Equity and Policy, Cambridge : MIT joint programme on the science and policy of global change, Report 27. 
Rose A. (1992). « Equity Considerations of Tradeable Carbon Entitlements ", in : S. Barrett, M. Grubb, K. Roland, A. Rose, R. Sandor, and T. Tietenberg, Combatting Global Warming : a Global System of Tradeable Carbon Emission Entitlements, UNCTAD, Genève.

Schelling T. (1992). "Some Economics of Global Warming ", American Economic Review, vol. 82, n 1, pp. $1-14$.

Sen A. (1993). Ethique et économie, Paris : PUF.

United Nations Framework Convention on Climate Change (UNFCCC) (1992). New York, May 9.

United Nations Framework Convention on Climate Change (UNFCCC) (1997). Kyoto Protocol to the United Nations Framework Convention on Climate Change.

Van Parijs P. (1991). Qu'est-ce qu'une sociétéjuste?, Paris : Seuil.

Van Parijs P. (1998). " Inégalité, justice et protection sociales ", Problèmes économiques, $\mathrm{n}^{\circ}$ 2.565-2.566, pp. 88-89.

Viguier L. (1999). L'environnement en économie communiste et post-communiste : de la crise systémique à l'agenda global, thèse de Doctorat, Université Pierre Mendès-France, Grenoble.

Young H. P., Wolf A. (1991). « Global Warming Negotiations : does Fairness Count?», Brookings Review, vol. $10, \mathrm{n}^{\circ} 2$, pp. $46-51$.
Annexe : liste des pays (ou régions) par catégories

Catégorie 1 : respect des objectifs fixés dans le Protocole de Kyoto

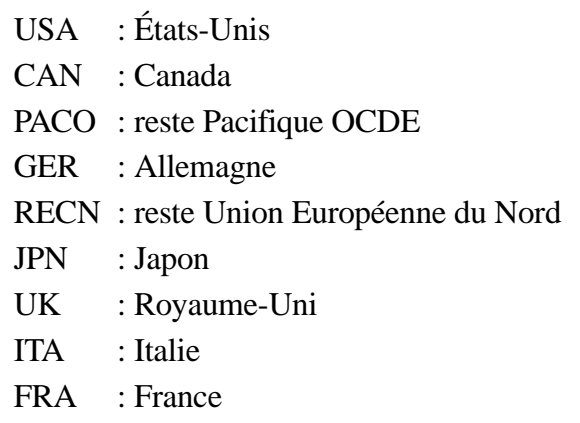

Catégorie 2 : respect des objectifs fixés dans le Protocole de Kyoto (pays annexe B), stabilisation des émissions en 2015 (pays non-annexe B)

CEU4 : 4 pays d'Europe Centrale (Hongrie, Pologne, République Tchèque, Slovaquie) (annexe B)

RECS : reste Union Européenne du Sud (annexe B)

KOR : Corée du Sud (non-annexe B)

Catégorie 3 : respect des objectifs fixés dans le Protocole de Kyoto (pays annexe $B$ ), stabilisation des émissions en 2030 (pays non-annexe $B$ )

MIEA : Moyen-Orient

FSUN : ex URSS

RCEU : reste Europe Centrale non-Annexe B

MEX : Mexique

CHN : Chine

OWEU : autres pays d'Europe de l'Ouest

RCAM : reste Amérique Centrale

BRA : Brésil

RSAM : reste Amérique du Sud

NOAF : Afrique du Nord

RSEA : reste Asie du Sud-Est

Catégorie 4 : stabilisation des émissions en 2045

SSAF : Afrique Sub-Saharienne

RSAS : reste Asie du Sud

NDE : Inde 